\title{
SUGAWARA CONSTRUCTION AND CASIMIR OPERATORS FOR KRICHEVER-NOVIKOV ALGEBRAS
}

\author{
Martin Schlichenmaier* And Oleg K. Sheinman*
}

December 95

\begin{abstract}
We show how to obtain from highest weight representations of KricheverNovikov algebras of affine type (also called higher genus affine Kac-Moody algebras) representations of centrally extended Krichever-Novikov vector field algebras via the Sugawara construction. This generalizes classical results where one obtains representations of the Virasoro algebra. Relations between the weights of the corresponding representations are given and Casimir operators are constructed. In an appendix the Sugawara construction for the multi-point situation is done.
\end{abstract}

\section{INTRODUCTION}

The Sugawara construction is one of the basic constructions in two-dimensional conformal field theory. Also from the point of view of representation theory it is at least for two reasons of importance. First, it provides a realization of highest weight representations of Virasoro-type algebras and second, the same technique is used for the investigation of Casimir operators for affine type algebras. Nevertheless, in the mathematical literature there is no consecutive presentation of this construction for higher genera with all necessary proofs. We hope that this article will fill this gap. Almost all necessary ideas are contained in Kac [8] (see also the references therein), Krichever and Novikov [10] and Bonora et al. [1] but each of these three treatments is too restrictive with respect to the genus, or to the properties of the underlying finite dimensional Lie

1991 Mathematics Subject Classification. 17B66, 17B67, 17B90, 30F30, 14H55, 81R10, 81T40.

Key words and phrases. Sugawara construction, highest weight representations, infinite-dimensional Lie algebras, central extensions, current algebras, Casimir operators.

*Partially supported by the RiP program of the Volkswagen-Stiftung. 
algebra, or at least to the completeness of the presentation. We give in this article a survey of the basic results in form of a detailed proof. In addition, we demonstrate the application of this technique in the construction of Casimir operators for higher genera and generalize it to the case of Riemann surfaces with many punctures.

In the framework of conformal field theory representations of affine Kac-Moody algebras and of the Virasoro algebra play a fundamental role. These algebras and their representations are also from the mathematical point of view of great interest. They give interesting examples of infinite dimensional Lie algebras which one still can handle. For their representations one has developed a fairly complete but nevertheless nontrivial structure theory. Another very fascinating aspect of them is that they have been proven to be very useful in proving deep mathematical results. Let us mention here the explanation of the "monster and moonshine" which relates the dimensions of the irreducible representations of the monster group with the coefficients of the $q$-expansion series for the elliptic modular function $j(q)$, observed by Conway and explained by Frenkel, Lepowski and Meurman [5] (see also Borcherds [2]). Recall that the monster is the largest exceptional finite simple group. There are lots of other important applications, like in the theory of integrable systems and so on.

Let us recall the definitions of these algebras. For details see [7], or [8] for a more pedagogical treatment. Let $\mathfrak{g}$ be a finite dimensional Lie algebra over $\mathbb{C}$. The current algebra (or loop algebra) is defined to be $\mathcal{G}=\mathfrak{g} \otimes \mathbb{C}\left[z, z^{-1}\right]$ as vector space. It is generated by the elements $x \otimes z^{n}$ with $n \in \mathbb{Z}, x \in \mathfrak{g}$ and Lie structure given by

$$
\left[x \otimes z^{n}, y \otimes z^{m}\right]=[x, y] \otimes z^{n+m} .
$$

If $\mathfrak{g}$ admits an invariant symmetric non-degenerate bilinear form (.....) then the affine (untwisted) Kac-Moody algebra is the centrally extended algebra $\widehat{\mathcal{G}}$ of the current algebra. As vector space $\widehat{\mathcal{G}}=\mathcal{G} \oplus \mathbb{C} t$ with Lie structure

$$
[x(n), y(m)]=[x, y](n+m)+(x \mid y) \cdot n \cdot \delta_{-m}^{n} \cdot t, \quad[t, \widehat{\mathcal{G}}]=0,
$$

where we used the usual notation $x(n):=x \otimes z^{n}$. Special cases are the situation where $\mathfrak{g}$ is simple and one takes the Cartan-Killing form or where $\mathfrak{g}$ is abelian and one takes an arbitrary non-degenerate symmetric bilinear form. In the latter case one calls $\widehat{\mathcal{G}}$ also a Heisenberg algebra.

Often it is convenient to adjoin an additional element $d$, a derivation, to $\widehat{\mathcal{G}}$ and obtain $\widehat{\mathcal{G}}^{d}:=\widehat{\mathcal{G}} \oplus \mathbb{C} d$ with Lie structure

$$
[d, t]=0, \quad[d, x(n)]=n \cdot x(n) .
$$

The Virasoro algebra $\widehat{\mathcal{L}}$ is the Lie algebra with basis $\left\{L_{n} \mid n \in \mathbb{Z}\right\} \cup\left\{t_{1}\right\}$ and Lie structure

$$
\left[L_{n}, L_{m}\right]=(m-n) L_{m+n}+\delta_{-n}^{m} \frac{n^{3}-n}{12} \cdot c \cdot t_{1}, \quad\left[L_{n}, t_{1}\right]=0
$$


The number $\mathrm{c}$ is sometimes called central charge. We denote the Lie algebra without the element $t_{1}$ by $\mathcal{L}$.

Note that all these algebras are $\mathbb{Z}$-graded algebras if one defines $\operatorname{deg}(x(n))=n$, $\operatorname{deg}\left(L_{n}\right)=n$, and $\operatorname{deg}(t)=\operatorname{deg}\left(t_{1}\right)=\operatorname{deg}(d)=0$. By the gradedness all above algebras split into subalgebras generated by the elements of negative, zero, resp. positive degree, e.g. $\widehat{\mathcal{G}}=\widehat{\mathcal{G}}_{-} \oplus \widehat{\mathcal{G}}_{0} \oplus \widehat{\mathcal{G}}_{+}$. The finite dimensional algebra $\mathfrak{g}$ can be embedded via $x \mapsto x(0)$ into $\widehat{\mathcal{G}}$ and one obtains $\widehat{\mathcal{G}}_{0} \cong \mathfrak{g} \oplus \mathbb{C} t$. This decomposition allows to define highest weight representations. These are representations $V$ which are generated by one element $\psi$ with $\mathcal{G}_{+} \psi=0$ and where the Cartan and the positive nilpotent subalgebras of $\widehat{\mathcal{G}}_{0}$ operate in a certain manner. In particular we get for every $v \in V$ and every $x \in \mathfrak{g}$ that $x(n) v=0$ for $n$ big enough. The similar construction works for the Virasoro algebra.

Indeed both algebras are related via the Sugawara construction. Let $V$ be a highest weight representation of $\widehat{\mathcal{G}}$, where $\mathfrak{g}$ is a simple Lie algebra, $\left\{u_{i}, i=1, \ldots, \operatorname{dim} \mathfrak{g}\right\}$ is a basis and $\left\{u^{i}\right\}$ is the dual basis. Let $k$ be the dual Coxeter number (see Section 3 for a description) and suppose that $t$ operates as $c \cdot i d$ on $V$ and that $k+c \neq 0$ then

$$
S_{k}:=-\frac{1}{2(k+c)} \sum_{n} \sum_{i}: u_{i}(-n) u^{i}(n+k):
$$

is a well-defined operator. Here $u_{i}(n)$ is considered as operator on $V$ and $: . . .$. denotes a normal ordering. The normal ordering takes care that the elements of highest degree are moved to the right where they eventually annihilate every fixed vector. What is quite astonishing is the fact that the map $L_{k} \mapsto S_{k}$ and $t_{1} \mapsto i d$ defines a representation of the Virasoro algebra with central charge $\frac{c \cdot \operatorname{dim} \mathfrak{g}}{c+k}$.

The highest weight of the Sugawara representation we can be read off the relation

$$
-2(\mathrm{k}+\mathrm{c}) \cdot S_{0} \psi=(\lambda+2 \bar{\rho} \mid \lambda) \psi
$$

where $\lambda$ is the highest weight of the $\widehat{\mathcal{G}}^{d}$-module from which we started and $\bar{\rho}$ is the half-sum of the positive roots of the Lie algebra $\mathfrak{g}$.

The above definitions look rather formal. But they have their geometric interpretation. The associative algebra $\mathbb{C}\left[z, z^{-1}\right]$ of Laurent polynomials can be identified with the algebra consisting of meromorphic functions on the Riemann sphere $\widehat{\mathbb{C}}=\mathbb{C} \cup\{\infty\}$ (with quasi-global coordinate $z \in \mathbb{C}$ ) which are holomorphic outside 0 and $\infty$. In this picture $\mathcal{G}$ is identified with the space of $\mathfrak{g}$-valued meromorphic functions on $\widehat{\mathbb{C}}$ obeying the same regularity condition. The algebra $\mathcal{L}$ can be interpreted as the Lie algebra of meromorphic vector fields on $\widehat{\mathbb{C}}$, again with this regularity condition, if we identify $L_{n}$ with $z^{n+1} \frac{d}{d z}$. In this description it is natural to ask for generalization to the case of compact Riemann surfaces $\Gamma$ of arbitrary genus. Essentially this generalization was done 
by Krichever and Novikov in [10]. Their main reason was that in the usual quantization of two-dimensional conformal field models by means of the Virasoro algebra the role played by the underlying Riemann surface is not clear. They fixed two points $P_{+}$and $P_{-}$and considered the algebra (resp. Lie algebra) of meromorphic functions (resp. vector fields) which are holomorphic outside the two points. (Indeed it is possible to consider the more general algebra of differential operators [17].) To obtain central extensions they gave a geometric definition for the defining cocycles. Using this approach they were able to create a far developed approach to the quantization on Riemann surfaces.

An essential step in the $g=0$ case was to introduce a graded structure for these algebras. This is not possible for higher genus. Fortunately in most cases what is needed is a weaker concept, an almost-graded structure. Krichever and Novikov did this by exhibiting a certain basis, indexed by the integers, and defining the basis elements to be the homogeneous elements. These basis elements will fulfil important duality relations.

We will describe this set-up in Section 2. Generalized affine Kac-Moody algebras were introduced by Krichever and Novikov [10], [11] and extensively studied by Sheinman [18], [19], [20] (see also Bremner [3],[4] and Jaffe, Klimek and Lesniewski [6]). For a multi-point generalization see Schlichenmaier [16], [17].

As explained above one obtains in the classical case from such a representation by the Sugawara construction a representation of the Virasoro algebra (with central extension). Our aim is to generalize this to higher genus with the goal to obtain representations of a centrally extended Krichever-Novikov vector field algebra. This we will do in Section 3, where the main result is Theorem 3.1. For $\mathfrak{g}$ an abelian Lie algebra this has been done by Krichever and Novikov [10]. In particular they made use of their beautiful technique of delta-distributions on Riemann surfaces. In the nonabelian case there is one important point namely the appearance of the dual Coxeter number in the final answer (see Theorem 3.1 below). It is necessary to use earlier ideas of [8] in order to explain this point in the framework of the Krichever-Novikov approach. For $\mathfrak{g}$ a simple Lie algebra the result has been stated by Bonora, Rinaldi, Russo and $\mathrm{Wu}$ in [1]. There also a sketch of a proof is given. The presentation there might not fulfil every requirement of a scrupulous mathematician on a proof. Especially if one sees what delicate questions on normal ordering are involved. For the above mentioned reasons and because in the appendix we want to generalize the construction to the multi-point situation we present here a complete proof (maybe we are following their line of arguments). By this we hope to convince also a sceptical mathematician that the result of Bonora and collaborators is correct. Again we obtain that the rescaled modes of the Sugawara operator of these representations will be a representation of a certain (explicitly given) central extension of the vector field algebra with the same central charge as in the genus zero case. A key step in the proof, which is an important result by its own, is

$$
\left[S_{k}, x(n)\right]=x\left(\nabla_{e_{k}} A_{n}\right)
$$


where $\nabla_{e_{k}} A_{n}$ is the function obtained by applying the Lie derivative with respect to the vector field $e_{k}$ to the function $A_{n}$, where the index denotes the Krichever-Novikov degree (see Prop. 3.1 and Prop. 3.2). This specializes for $g=0$ to $\left[S_{k}, x(n)\right]=n x(n+k)$.

Some of the proofs will be postponed to Section 4 which is in some sense of more technical nature. An important tool in the proof will be the duality property of the Krichever-Novikov basis and the "delta distribution", see (2-19). Nevertheless we prove there also very astonishing identities between numbers which are obtained with the help of meromorphic forms of different weights. Up to now, we do not understand these identities completely.

In Section 5 we recall the notion of the weight of a representation of the KricheverNovikov algebras of affine type (generalized affine Kac-Moody algebras) and of the Krichever Novikov vector field algebra as introduced in [19]. We show that with respect to the Sugawara construction on Riemann surfaces the weights are related in a way similar to (1-6). Additionally, structure constants and the cocycle of the algebra of meromorphic functions $\left\{A_{n}\right\}$ are involved (see Theorem 5.1).

In Section 6 we add a vector field $e$ to $\widehat{\mathcal{G}}$ that generalizes the adjoining of the derivation $d$ in the classical case. Let us denote the obtained Lie algebra by $\widehat{\mathcal{G}}^{e}$. We construct higher genus Casimir operators. If the vector field $e$ is given as a certain linear combination of the basis $e_{k}$ introduced by Krichever and Novikov, then the Casimir operator $\Omega$ is given as $2 L+2(\mathrm{c}+\mathrm{k}) e$, where $L$ is a linear combination of the (not rescaled) Sugawara operators $L_{k}$ with the same coefficients as in the combination of $e$. Furthermore, under the hypothesis that the Casimir operator admits an eigenvector we calculate the eigenvalue in terms of the weight $\Lambda$ calculated in Section 5 and the highest weight of the vector field $e$ in the corresponding $\widehat{\mathcal{G}}^{e}$-module.

The Sugawara construction can be generalized to the situation where one allows poles at more than two points. This will be done in Appendix A. The crucial step for this more general set-up is to introduce an almost-grading and to find dual systems of basis elements. This is done in [16], [15, 3.ref.]. For a quick review see [17]. (In this context see also Sadov [13] and the appendix of [12].)

The authors gratefully acknowledge the Volkswagen-Stiftung for their support in the Research-in-Pairs program. We would also like to thank the Mathematisches Forschungsinstitut in Oberwolfach for its hospitality while preparing this article. 


\section{The General Set-UP}

In this section we would like to recall the necessary facts on the global KricheverNovikov approach to conformal field theory. For the following let $\Gamma$ be a compact Riemann surface of arbitrary genus $g, P_{+}$and $P_{-}$two fixed points which for genus $g \geq 1$ are in general position. Let $\Gamma^{*}=\Gamma \backslash\left\{P_{+}, P_{-}\right\}$, and let $\rho$ be the unique meromorphic differential with exact pole order 1 at the points $P_{ \pm}$and residues $\operatorname{res}_{P_{ \pm}}(\rho)= \pm 1$, holomorphic elsewhere and with purely imaginary periods. We fix a point $Q \in \Gamma^{*}$. The function $u(P)=\operatorname{Re} \int_{Q}^{P} \rho$ is a well-defined harmonic function. The level lines

$$
C_{\tau}=\left\{P \in \Gamma^{*} \mid u(P)=\tau\right\}, \quad \tau \in \mathbb{R}
$$

define a fibering of $\Gamma^{*}$. For $\tau \ll 0(\tau \gg 0)$ the level line $C_{\tau}$ is a deformed cycle around $P_{+},\left(\right.$resp. $\left.P_{-}\right)$.

Let $K$ be the canonical bundle, i.e. the bundle whose local sections are the local holomorphic differentials. For every $\lambda \in \mathbb{Z}$ we consider the bundle $K^{\lambda}:=K^{\otimes \lambda}$, the bundle with local sections the forms of weight $\lambda$. (After fixing a square root of the canonical bundle, a so-called theta characteristic, it is possible to deal with $\lambda \in \frac{1}{2} \mathbb{Z}$.) We denote by $\mathcal{F}^{\lambda}$ the vector space of global meromorphic sections of $K^{\lambda}$ which are holomorphic on $\Gamma^{*}$. Special cases are the differentials $(\lambda=1)$, the functions $(\lambda=0)$, and the vector fields $(\lambda=-1)$. To denote the space of functions we use also $\mathcal{A}$, for the space of vector fields we use also $\mathcal{L}$.

The (associative) algebra of functions $\mathcal{A}$ operates by multiplication on $\mathcal{F}^{\lambda}$. The vector fields (i.e. the elements in $\mathcal{L}$ ) operate by taking the Lie derivative on $\mathcal{F}^{\lambda}$. In local coordinates the Lie derivative can be described as

$$
\nabla_{e}(g)_{\mid}=\left(e(z) \frac{d}{d z}\right) \cdot\left(g(z) d z^{\lambda}\right)=\left(e(z) \frac{d g}{d z}(z)+\lambda g(z) \frac{d e}{d z}(z)\right) d z^{\lambda} .
$$

Here and in the following we will use the same symbol for the section of the bundle and its local representing function. By $(2-2) \mathcal{L}$ becomes a Lie algebra and the vector spaces $\mathcal{F}^{\lambda}$ become Lie modules over $\mathcal{L}$ (i.e. we have $\left.\left[\nabla_{e}, \nabla_{f}\right]=\nabla_{[e, f]}\right)$.

Note that in the case $g=0$ with quasi-global coordinate $z$ and $P_{+}=\{z=0\}$ and $P_{-}=\{z=\infty\}$ we obtain $\mathcal{A}=\mathbb{C}\left[z, z^{-1}\right]$, the algebra of Laurent polynomials and for $\mathcal{L}$ the Witt-Algebra, the Lie algebra with basis $\left\{l_{n}=z^{n+1} \frac{d}{d z} \mid n \in \mathbb{Z}\right\}$ and the commutator relation $\left[l_{n}, l_{m}\right]=(m-n) l_{m+n}$. We will call this case the "the classical case".

For the following let $\mathfrak{g}$ be a finite dimensional reductive Lie algebra, i.e. a direct sum of an abelian and a semi-simple Lie algebra, and let (..|..) be a non-degenerate symmetric invariant bilinear form on $\mathfrak{g}$. In particular we have $([x, y] \mid z)=(x \mid[y, z])$. 
We can take the Cartan-Killing form in the semi-simple case and any non-degenerate symmetric form in the abelian case. The algebra $\mathcal{G}=\mathfrak{g} \otimes \mathcal{A}$ is called the current algebra. It can be considered as the algebra of $\mathfrak{g}$-valued meromorphic functions on $\Gamma$ which are holomorphic on $\Gamma^{*}$.

We take an element $\alpha \in H_{1}\left(\Gamma^{*}, \mathbb{Z}\right)$ and represent it by a cycle $Z_{\alpha}$ which is a sum of differentiable curves on $\Gamma$. The map

$$
\gamma_{\alpha}: \mathcal{A} \times \mathcal{A} \rightarrow \mathbb{C}, \quad \gamma_{\alpha}(f, g):=\frac{1}{2 \pi \mathrm{i}} \int_{Z_{\alpha}} f d g
$$

defines a 2 -cocycle for the abelian Lie algebra $\mathcal{A}$. Due to the fact that all poles are located in $P_{ \pm}$homologous cycles $Z_{\alpha}$ define the same cocycle $\gamma_{\alpha}$. Using the cocycle (2-3) a central extension $\widehat{\mathcal{A}}_{\alpha}$ is obtained: $0 \longrightarrow \mathbb{C} \longrightarrow \widehat{\mathcal{A}}_{\alpha} \longrightarrow \mathcal{A} \longrightarrow 0$. If $\hat{f}$ and $\hat{g}$ are lifts of elements $f, g \in \mathcal{A}$ and $t$ is a generator of the center then

$$
[\hat{f}, \hat{g}]=-\gamma_{\alpha}(f, g) \cdot t, \quad\left[t, \widehat{\mathcal{A}}_{a}\right]=0
$$

The algebra $\widehat{\mathcal{A}}_{\alpha}$ is called a Heisenberg algebra (of higher genus). This can be generalized to the current algebra. We take $\widehat{\mathcal{G}}_{\alpha}=\mathcal{G} \oplus \mathbb{C} \cdot t$ as vector space and define the Lie algebra structure as follows. For $a \in \mathcal{G}$ we use $\hat{a}=(a, 0)$ for the corresponding lift in $\widehat{\mathcal{G}}_{a}$ and define for $x, y \in \mathfrak{g}$

$$
[\widehat{x \otimes f}, \widehat{y \otimes g}]=[x, \widehat{y] \otimes(} f g)-(x \mid y) \cdot \gamma_{\alpha}(f, g) \cdot t, \quad[t, \widehat{\mathcal{G}} \alpha]=0 .
$$

From the invariance of the bilinear form (..|..) it follows that this is indeed a central extension of $\mathcal{G}$. The algebras obtained in this way are called Krichever-Novikov algebras of affine type. They are higher genus generalizations of affine Kac-Moody algebra. In Section 6 we will adjoin an additional vector field to the algebra $\widehat{\mathcal{G}}_{\alpha}$.

In the following an important role will be played by the central extensions corresponding to the cycle which is represented by a level line $C_{\tau}$. If we use $\gamma_{0}$ or just $\gamma$ we will always mean this 2 -cocycle. And we will denote by $\widehat{\mathcal{A}}$ and $\widehat{\mathcal{G}}$ the corresponding central extensions.

In this article we also have to deal with central extensions of the vector field algebra. Krichever and Novikov gave a generalization of the standard cocycle of the Virasoro algebra to higher genus as follows. Let $R$ be a projective connection (holomorphic or meromorphic with poles only at $P_{ \pm}$) on $\Gamma$. For the definition of a projective connection see [10]. Note that the difference of two projective connections is a form of weight 2 (i.e. a quadratic differential).

For vector fields $e$ and $f$ represented locally as $e(z) \frac{d}{d z}$ and $f(z) \frac{d}{d z}$ the 2-cocycle is defined as

$$
\chi_{\alpha, R}(e, f)=\frac{1}{24 \pi \mathrm{i}} \int_{Z_{\alpha}}\left(\frac{1}{2}\left(e^{\prime \prime \prime} f-e f^{\prime \prime \prime}\right)-R \cdot\left(e^{\prime} f-e f^{\prime}\right)\right) d z
$$


Without the connection $R$ the expression under the integral would not be a well-defined differential. The choice of a different projective connection will yield a cohomologous cocycle. The central extension is given by $\widehat{\mathcal{L}}_{\alpha}=\mathcal{L} \oplus \mathbb{C} \cdot t$ as vector space with Lie structure (using $\hat{e}=(e, 0)$ )

$$
[\widehat{e}, \widehat{f}]=\widehat{[e, f]}+\chi_{\alpha}(e, f) \cdot t, \quad[t, \widehat{\mathcal{L}}]=0
$$

Again we use $\widehat{\mathcal{L}}=\widehat{\mathcal{L}}_{0}=\widehat{\mathcal{L}}_{\left[C_{\tau}\right]}$ if we integrate over a level line. There is also a suitable extension to the multi-point situation (see Appendix A, [16] and the third article in Ref. $[15])$.

For the construction of highest weight representations in the classical case it is important that the above algebras are graded algebras. Note that in this case there is only one cycle class, hence only one nontrivial central extension (up to equivalence and isomorphy) defined as above. In the higher genus it is the concept of almost-grading which will do the job. Such a grading has been introduced by Krichever and Novikov in the following way. Let $g=0$ and $\lambda \in \mathbb{Z}$ be arbitrary or $g \geq 2$ and $\lambda \neq 0,1$ then there is for every $n \in \mathbb{Z}$ a unique (up to multiplication with a scalar) $f_{n}^{\lambda} \in \mathcal{F}^{\lambda}$ such that

$$
\operatorname{ord}_{P_{+}}\left(f_{n}^{\lambda}\right)=n-\lambda, \quad \operatorname{ord}_{P_{-}}\left(f_{n}^{\lambda}\right)=-n+(\lambda-1)+(2 \lambda-1)(g-1) .
$$

If we fix a local coordinate $z_{+}$at $P_{+}$we adjust the scalar by requiring locally

$$
f_{n}^{\lambda}\left(z_{+}\right)_{1}=z_{+}^{n-\lambda}\left(1+O\left(z_{+}\right)\right) d z^{\lambda}
$$

Note that our indexing differs from the one used by Krichever and Novikov by a shift. The set $\left\{f_{n}^{\lambda} \mid n \in \mathbb{Z}\right\}$ is a basis of $\mathcal{F}^{\lambda}$. By calculation of residues at $P_{ \pm}$we obtain

$$
\frac{1}{2 \pi \mathrm{i}} \int_{C_{\tau}} f_{n}^{\lambda} \cdot f_{m}^{1-\lambda}=\delta_{n,-m}
$$

It is quite convenient to introduce the notation $f_{\lambda}^{*, n}=f_{-n}^{\lambda}, A_{n}=f_{n}^{0}, e_{n}=f_{n}^{-1}, \omega^{n}=$ $f_{1}^{*, n}, \Omega^{n}=f_{2}^{*, n}$. Now the duality reads as

$$
\frac{1}{2 \pi \mathrm{i}} \int_{C_{\tau}} A_{n} \omega^{m}=\delta_{n}^{m}, \quad \frac{1}{2 \pi \mathrm{i}} \int_{C_{\tau}} e_{n} \Omega^{m}=\delta_{n}^{m}
$$

For the remaining cases of $\lambda$ and $n$ we have to modify the above prescription for $-g \leq$ $n \leq 0$. We set $A_{0}=1$ and $\omega^{0}=\rho$ and fix the elements $A_{n}$ and $\omega^{n}$ for $-g \leq n \leq-1$ by

$$
\begin{array}{ll}
\operatorname{ord}_{P_{+}}\left(A_{n}\right)=n, & \operatorname{ord}_{P_{-}}\left(A_{n}\right)=-n-g-1, \\
\operatorname{ord}_{P_{+}}\left(w^{n}\right)=-n-1, & \operatorname{ord}_{P_{-}}\left(w^{n}\right)=n+g
\end{array}
$$


and the duality (2-11). In all cases we obtain the same order at the point $P_{+}$and the same duality relation as in the generic case. We will use the term critical strip to denote the index values $-g, \ldots,-1,0$.

We define $\operatorname{deg} f_{n}^{\lambda}:=n$ and call this elements homogeneous elements of degree $n$. With respect to this degree the algebras $\mathcal{A}, \mathcal{L}, \mathcal{G}$ are almost-graded (where in the latter case we define for $\left.x \in \mathfrak{g}, \operatorname{deg}\left(x \otimes A_{n}\right):=n\right)$ and the vector spaces $\mathcal{F}^{\lambda}$ are almost-graded modules over $\mathcal{A}$ and $\mathcal{L}$. More precisely, we have

$$
A_{n} \cdot A_{m}=\sum_{k=n+m}^{n+m+L} \alpha_{n m}^{k} A_{k}, \quad\left[e_{n}, e_{m}\right]=\sum_{k=n+m}^{n+m+M} C_{n m}^{k} e_{k},
$$

where the constants are given by the duality relations as

$$
\alpha_{n m}^{k}=\frac{1}{2 \pi \mathrm{i}} \int_{C_{\tau}} A_{n} A_{m} \omega^{k}, \quad C_{n m}^{k}=\frac{1}{2 \pi \mathrm{i}} \int_{C_{\tau}}\left(\left[e_{n}, e_{m}\right]\right) \cdot \Omega^{k},
$$

and the constants $L$ and $M$ do not depend on $n$ and $m$. For $g \neq 1$ we calculate $M=3 g$. For $g=0$ we obtain $L=0$. In general explicit formulas can be given. For us of importance is only that if $n$ and $m$ are both on the same side of the critical strip (e.g. $n, m<-g$ ) then the upper bound will be $n+m+g$.

We want to extend our almost-grading to the central extensions $\widehat{\mathcal{A}}_{\alpha}, \widehat{\mathcal{L}}_{\alpha}, \widehat{\mathcal{G}}_{\alpha}$ by defining $\operatorname{deg} \widehat{x}:=\operatorname{deg} x$ and $\operatorname{deg} t:=0$. For this to work our 2-cocycles which define the central extensions should be local cocycles [10], i. e. there should be constants $K$ and $N$, such that

$$
\gamma_{\alpha}\left(A_{n}, A_{m}\right)=0, \quad \text { for }|n+m|>K, \quad \chi_{\alpha}\left(e_{n}, e_{m}\right)=0, \quad \text { for }|n+m|>N .
$$

For arbitrary $\alpha \in H_{1}\left(\Gamma^{*}, \mathbb{Z}\right)$ this will not be the case. But if we integrate over a level line $C_{\tau}$ we get that $\gamma\left(A_{n}, A_{m}\right) \neq 0$ implies $-2 g-2 \leq n+m \leq 0$ (for generic $n$ and $m$ we get as lower bound even $-2 g)$ and that $\chi\left(e_{n}, e_{m}\right) \neq 0$ implies $-6 g \leq n+m \leq 0$ (for $g \neq 1$ ). For later reference we note that at the upper boundary we obtain

$$
\gamma\left(A_{n}, A_{-n}\right)=(-n), \quad \chi\left(e_{n}, e_{-n}\right)=\frac{1}{12}\left(n^{3}-n\right) .
$$

We consider the vector space decomposition

$$
\begin{gathered}
\mathcal{A}=\mathcal{A}_{-} \oplus \mathcal{A}_{0} \oplus \mathcal{A}_{+}, \quad \text { with } \\
\mathcal{A}_{-}:=\left\langle A_{n} \mid n \leq-g-1\right\rangle, \quad \mathcal{A}_{0}:=\left\langle A_{n} \mid-g \leq n \leq 0\right\rangle, \quad \mathcal{A}_{+}:=\left\langle A_{n} \mid n \geq 1\right\rangle,
\end{gathered}
$$

and the corresponding decomposition

$$
\mathcal{G}=\mathcal{G}_{-} \oplus \mathcal{G}_{0} \oplus \mathcal{G}_{+}, \quad \text { with } \quad \mathcal{G}_{\beta}=\mathfrak{g} \otimes \mathcal{A}_{\beta}, \quad \beta \in\{-, 0,+\} .
$$


Due to the almost-gradedness of $\mathcal{A}$ we see that $\mathcal{A}_{+}$and $\mathcal{A}_{-}$, resp. $\mathcal{G}_{+}$and $\mathcal{G}_{-}$are subalgebras. Contrary to the classical case for higher genus $\mathcal{A}_{0}$ and $\mathcal{G}_{0}$ will only be subspaces. We will call the elements of $\mathcal{A}_{0}$ also the elements from the critical strip. By the locality of the cocycle this decomposition extends to $\widehat{\mathcal{G}}=\widehat{\mathcal{G}}_{-} \oplus \widehat{\mathcal{G}}_{0} \oplus \widehat{\mathcal{G}}_{+}$, where $\widehat{\mathcal{G}}_{ \pm}$can be identified with $\mathcal{G}_{ \pm}$and $\widehat{\mathcal{G}}_{0}=\mathcal{G}_{0} \oplus \mathbb{C} \cdot t$. Clearly $\widehat{\mathcal{G}}$ is generated by $\widehat{x \otimes A_{n}}$ for $x \in \mathfrak{g}$ and $n \in \mathbb{Z}$ and by the central element $t$. We will denote the element $\widehat{x \otimes A_{n}} \in \widehat{\mathcal{G}}$ and $x \otimes A_{n} \in \mathcal{G}$ also by $x(n)$ if convenient. Note that for $g=0$ we have $A_{n}=z^{n}$ and our notation specializes completely to the classical notation.

We close this section by introducing the very useful object

$$
\Delta\left(Q^{\prime}, Q\right)=\sum_{n \in \mathbb{Z}} A_{n}\left(Q^{\prime}\right) \omega^{n}(Q)
$$

It can be considered as the delta distribution in the sense that we have for $f \in \mathcal{A}$ and $\omega \in \mathcal{F}^{1}$

$$
\frac{1}{2 \pi \mathrm{i}} \int_{C_{\tau}} \Delta\left(Q^{\prime}, Q\right) f(Q)=f\left(Q^{\prime}\right), \quad \text { resp. } \quad \frac{1}{2 \pi \mathrm{i}} \int_{C_{\tau^{\prime}}} \Delta\left(Q^{\prime}, Q\right) \omega\left(Q^{\prime}\right)=\omega(Q) .
$$

Of course (2-19) can be extended to arbitrary pairs of weights $(\lambda, 1-\lambda)$.

\section{The Sugawara Construction}

Definition. A module $V$ over the Lie algebra $\widehat{\mathcal{G}}$ (resp. a representation) is called an admissible module (resp. representation) if for every $v \in V$ and for all $x \in \mathfrak{g}$ we have $x(n) v=0$ for $n \gg 0$.

Let $V$ be a fixed admissible module. Let the central element $t$ operate by multiplication with a scalar $c \in \mathbb{C}$. In the classical case these are the usual highest weight modules of affine Kac-Moody algebras. For higher genus such modules have been studied by Sheinman [18], [19], [20]. If $x \otimes A$ (or more precisely $\widehat{x \otimes A}$ ) is an element of $\widehat{\mathcal{G}}$ then we will use the notation $x(A)$ for the corresponding operator on $V$. For $x \otimes A_{n}$ with $A_{n}$ the special basis elements we will also use for short $x(n)$ to denote $x\left(A_{n}\right)$.

Recall that we assume $\mathfrak{g}$ to be a finite dimensional reductive Lie algebra. We choose a basis $u_{i}, i=1, \ldots, \operatorname{dim} \mathfrak{g}$ of $\mathfrak{g}$ and the corresponding dual basis $u^{i}, i=1, \ldots, \operatorname{dim} \mathfrak{g}$ with respect to the invariant non-degenerate symmetric bilinear form (..|..). The Casimir element $\Omega^{0}=\sum_{i=1}^{\operatorname{dim} \mathfrak{g}} u_{i} u^{i}$ of the universal enveloping algebra $U(\mathfrak{g})$ is independent of the choice of the basis. In the following a summation over $i$ is always assumed to be over the above summation range. 


\section{Lemma 3.1.}

$$
\left[\Omega^{0}, \mathfrak{g}\right]=0 .
$$

$$
\begin{aligned}
& \sum_{i}\left[u_{i}, u^{i}\right]=0 . \\
& \sum_{i}\left[u_{i} \otimes A_{n}, u^{i} \otimes A_{m}\right]=-\operatorname{dim} \mathfrak{g} \cdot \gamma\left(A_{n}, A_{m}\right) \cdot t
\end{aligned}
$$

(4) For $\mathfrak{g}$ an abelian or a simple Lie algebra there is a constant $\mathrm{k}$, such that $\sum_{i} a d_{u_{i}} \circ a d_{u^{i}}=2 \mathrm{k}$.

Proof. (1) and (4) are standard knowledge. (2) follows from the invariance of bases. To show (3) we take the structure equation of the centrally extended algebra

$$
\left[u_{i} \otimes A_{n}, u^{i} \otimes A_{m}\right]=\left[u_{i}, u^{i}\right] \otimes\left(A_{n} A_{m}\right)-\gamma\left(A_{n}, A_{m}\right) \cdot\left(u_{i} \mid u^{i}\right) \cdot t
$$

After summation over $i$ the first summand will vanish by (2) and we get the result.

Note that $2 k$ is the eigenvalue of the Casimir operator in the adjoint representation. In the case where $\mathfrak{g}$ is simple $\mathrm{k}$ is the dual Coxeter number. In the abelian case $k=0$. Let us define for $Q \in \Gamma$ the formal sum (the "generating function")

$$
\widehat{x}(Q)=\sum_{n} x(n) \cdot \omega^{n}(Q)
$$

Here and in the following a summation over the indices of our forms will always mean a summation over $\mathbb{Z}$ if not stated otherwise. We define the higher genus Sugawara (or Segal) operator

$$
T(Q):=\frac{1}{2} \sum_{i}: \widehat{u_{i}}(Q) \widehat{u^{i}}(Q):=\frac{1}{2} \sum_{n, m} \sum_{i}: u_{i}(n) u^{i}(m): \omega^{n}(Q) \omega^{m}(Q) .
$$

Here $: . . .$. : denotes some normal ordering. If we consider again $T(Q)$ as "generating function" we can write

$$
T(Q)=\sum_{k} L_{k} \cdot \Omega^{k}(Q)
$$

with certain operators $L_{k}$. Using duality we obtain

$$
\begin{gathered}
L_{k}=\frac{1}{2 \pi \mathrm{i}} \int_{C_{\tau}} T(Q) e_{k}(Q)=\frac{1}{2} \sum_{n, m} \sum_{i}: u_{i}(n) u^{i}(m): l_{k}^{n m}, \\
\text { with } \quad l_{k}^{n m}=\frac{1}{2 \pi \mathrm{i}} \int_{C_{\tau}} w^{n}(Q) w^{m}(Q) e_{k}(Q) .
\end{gathered}
$$


Note that for a fixed value of $k$ for every value of $n$ there is only a finite set of values for $m$ such that $l_{k}^{n m} \neq 0$. More precisely, $l_{k}^{n m} \neq 0$ implies that for the indices $n$ and $m$ outside the exceptional strip we have $k-n \leq m \leq k-n+g$. This specializes for $g=0$ to $l_{k}^{n m}=\delta_{k}^{m+n}$ which gives the usual definition of the $g=0$ Sugawara operators (see [8] and references therein). By this finiteness and the normal ordering the operators $L_{k} \in g l(V)$ are well-defined.

We will choose in Section 3 and Section 4 the prescription

$$
: x(n) y(m)::= \begin{cases}x(n) y(m) & , n \leq m \\ y(m) x(n) & , n>m\end{cases}
$$

as normal ordering. We will see in the proofs that Proposition 3.1 and hence Proposition 3.2 will not depend on the normal ordering. In Proposition 3.3 where we show that the Sugawara operators define a centrally extended Krichever-Novikov algebra we will see that only the cohomology class of the cocycle defining the central extension will depend on the normal ordering chosen.

The following proposition is the key step in the construction.

Proposition 3.1. Let $\mathfrak{g}$ be either an abelian or a simple Lie algebra, then

$$
\begin{gathered}
{\left[L_{k}, x(r)\right]=-(\mathrm{c}+\mathrm{k}) \sum_{v} K_{r, k}^{v} x(v)} \\
\text { with } K_{r, k}^{v}:=\frac{1}{2 \pi \mathrm{i}} \int_{C_{\tau}} w^{v}(Q) e_{k}(Q) d A_{r}(Q)=\sum_{m} l_{k}^{v m} \gamma_{m r}, \\
\gamma_{m r}:=\gamma\left(A_{m}, A_{r}\right):=\frac{1}{2 \pi \mathrm{i}} \int_{C_{\tau}} A_{m}(Q) d A_{r}(Q) .
\end{gathered}
$$

The result does not depend on the normal ordering.

Note that all the infinite sums above are indeed well-defined finite sums. The above proposition will specialise in the classical case to $\left[L_{k}, x(r)\right]=-(\mathrm{c}+\mathrm{k}) r x(r+k)$ (see [8, Prop.10.1]). We will postpone the proof to the next section. Here we want to show the relation (3-7). Using the "delta distribution" (2-19) we obtain

$$
\begin{gathered}
\sum_{m} l_{k}^{v m} \gamma_{m r}=\sum_{m} \frac{1}{2 \pi \mathrm{i}} \int_{C_{\tau}} \omega^{v}(Q) \omega^{m}(Q) e_{k}(Q) \frac{1}{2 \pi \mathrm{i}} \int_{C_{\tau^{\prime}}} A_{m}\left(Q^{\prime}\right) d A_{r}\left(Q^{\prime}\right)= \\
\frac{1}{(2 \pi \mathrm{i})^{2}} \iint_{C_{\tau}} \omega_{C_{\tau^{\prime}}}^{v}(Q) e_{k}(Q) d A_{r}\left(Q^{\prime}\right) \Delta\left(Q^{\prime}, Q\right)=\frac{1}{2 \pi \mathrm{i}} \int_{C_{\tau^{\prime}}} \omega^{v}\left(Q^{\prime}\right) e_{k}\left(Q^{\prime}\right) d A_{r}\left(Q^{\prime}\right)=K_{r, k}^{v} .
\end{gathered}
$$

We define the operation of $e \in \mathcal{L}$ on $\widehat{x}(Q)$ as

$$
e . \widehat{x}(Q):=\sum_{n} x(n)\left(\nabla_{e} \omega^{n}\right)(Q), \quad \text { for } \quad \widehat{x}(Q)=\sum_{n} x(n) \omega^{n}(Q) .
$$




\section{Proposition 3.2.}

$$
\begin{aligned}
& {\left[L_{k}, x(r)\right]=-(\mathrm{c}+\mathrm{k}) x\left(\nabla_{e_{k}} A_{r}\right) .} \\
& {\left[L_{k}, \widehat{x}(Q)\right]=(\mathrm{c}+\mathrm{k}) e_{k} \cdot \widehat{x}(Q) .}
\end{aligned}
$$

Proof. We can write $\nabla_{e_{k}} A_{r}$ in local coordinates as $e_{k}(z) \frac{d A_{r}(z)}{d z}$, hence

$$
\left(\nabla_{e_{k}} A_{r}\right)(Q)=e_{k}(Q) d A_{r}(Q)=\sum_{v} \beta_{r, k}^{v} A_{v}(Q)
$$

By duality the coefficients $\beta_{r, k}^{v}$ calculate as $\beta_{r, k}^{v}=\frac{1}{2 \pi \mathrm{i}} \int_{C_{\tau}} e_{k}(Q) d A_{r}(Q) \omega^{v}(Q)=K_{r, k}^{v}$. Hence Prop. 3.1 implies (1).

To prove (2) we write $\nabla_{e_{k}} \omega^{v}=\sum_{r} \zeta_{r, k}^{v} \omega^{r}$ where

$$
\zeta_{r, k}^{v}=\frac{1}{2 \pi \mathrm{i}} \int_{C_{\tau}}\left(\nabla_{e_{k}} \omega^{v}\right) A_{r}=\frac{1}{2 \pi \mathrm{i}} \int_{C_{\tau}} \nabla_{e_{k}}\left(\omega^{v} A_{r}\right)-\frac{1}{2 \pi \mathrm{i}} \int_{C_{\tau}} \omega^{v} \nabla_{e_{k}} A_{r}=-K_{r, k}^{v}
$$

because the residue of a Lie derivative of a meromorphic differential will vanish (see for example [16, p.102]). This proves (2).

Proposition 3.3. The operators $L_{k} \in g l(V)$ and $i d=1 \in g l(V)$ close up to a Lie subalgebra of $g l(V)$ with commutator relation

$$
\left[L_{k}, L_{l}\right]=-(\mathbf{c}+\mathrm{k}) \sum_{n} C_{k l}^{n} L_{n}-\frac{1}{2} \mathrm{c}(\mathbf{c}+\mathrm{k}) \operatorname{dim} \mathfrak{g} \cdot \chi_{k l} \cdot i d
$$

where $C_{k l}^{n}$ are the structure constants of the vector field algebra $\mathcal{L}$ and

$$
\begin{gathered}
\chi_{k l}=\psi_{k l}+\widehat{\chi}_{k l}, \quad \psi_{k l}=\sum_{s, v} \sum_{n=0}^{v+1} C_{k l}^{s} l_{s}^{n v} \gamma_{n v}=\sum_{v} \sum_{n=0}^{v+1} E_{k l}^{n v} \gamma_{n v}, \\
E_{k l}^{n v}=\frac{1}{2 \pi \mathrm{i}} \int_{C_{\tau}}\left[e_{k}, e_{l}\right] \cdot \omega^{n} \omega^{v}, \quad \widehat{\chi}_{k l}=\left(\sum_{\substack{n>0 \\
v \leq 0}}-\sum_{\substack{n \leq 0 \\
v>0}}\right) K_{v, k}^{n} K_{n, l}^{v} .
\end{gathered}
$$

The $\chi_{k l}$ could be non-zero only if $-6 g \leq k+l \leq 0$. A different normal ordering will change the range of the $n$ and $v$ summation in the definition of $\psi_{k l}$ and hence change $\chi_{k l}$. At the upper bound we obtain

$$
\chi_{k,-k}=-\frac{1}{6}\left(k^{3}-k\right) .
$$


If $\mathrm{c}+\mathrm{k} \neq 0$ we can choose the rescaled elements $L_{k}^{*}=\frac{-1}{\mathrm{c}+\mathrm{k}} L_{k}$ and obtain

$$
\left[L_{k}^{*}, L_{l}^{*}\right]=\sum_{n} C_{k l}^{n} L_{n}^{*}-\frac{\mathrm{c}}{2(\mathrm{c}+\mathrm{k})} \operatorname{dim} \mathfrak{g} \cdot \chi_{k l} \cdot i d
$$

Hence we obtain a representation of a centrally extended algebra of $\mathcal{L}$. Note that from the Jacobi identity inside $g l(V)$ and inside $\mathcal{L}$ it follows that $\chi_{k l}$ indeed defines a 2cocycle $\chi$ for the Lie algebra $\mathcal{L}$. By Proposition 3.3 this cocycle is local. Krichever and Novikov showed ([10], [11]) that all local cocycles are cohomologous to a multiple of the geometric cocycle $\chi_{R}(2-6)$ with a suitable projective connection $R$ (and integration over a level line $C_{\tau}$ ).

Hence the centrally extended algebra is a representation of the algebra $\widehat{\mathcal{L}}$. To study it in more detail the following facts are quite useful.

Lemma 3.2. Let $\chi_{R}$ be the cocycle (2-6) (where the integration curve equals $C_{\tau}$ ).

(a) $R$ is a meromorphic projective connection with only poles up to order two at the points $P_{ \pm}$if and only if $\chi_{R}\left(e_{k}, e_{l}\right)=0$ for $k+l>0$ or $k+l<-6 g$.

(b) Let $R_{\mid}\left(z_{+}\right)=\alpha_{+} z_{+}^{-2}\left(1+O\left(z_{+}\right)\right)$be the local form of the projective connection at the point $P_{+}$, then

$$
\chi_{R}\left(e_{k}, e_{-k}\right)=\frac{1}{12}\left(k^{3}-k-2 \alpha_{+} k\right)
$$

To show this one calculates the involved residues (see also [10]).

By Proposition 3.3 we see that $\chi$ is a cocycle which fulfils the conditions of Lemma 3.2(a) Hence $\chi=d \cdot \chi_{R}$ with $d \in \mathbb{C}$ and $R$ a suitable projective connection. If we compare $\chi_{R}\left(e_{k}, e_{-k}\right)$ and $\chi\left(e_{k}, e_{-k}\right)$ we see that $\alpha_{+}=0$ (hence $\left.\operatorname{ord}_{P_{+}}(R) \geq-1\right)$ and $d=-2$. Altogether we obtain the following theorem:

Theorem 3.1. Let $\mathfrak{g}$ be either a finite dimensional abelian or simple Lie algebra and $2 \mathrm{k}$ be the eigenvalue of the Casimir operator in the adjoint representation and $\widehat{\mathcal{G}}$ be the higher genus affine Kac-Moody algebra. Let $V$ be an admissible representation where the central element operates as $\mathrm{c} \cdot$ identity. If $\mathrm{c}+\mathrm{k} \neq 0$ then the rescaled modes

$$
L_{k}^{*}=\frac{-1}{2(\mathrm{c}+\mathrm{k})} \sum_{n, m} \sum_{i}: u_{i}(n) u^{i}(m): l_{k}^{n m}=\frac{-1}{2 \pi \mathrm{i}(\mathrm{c}+\mathrm{k})} \int_{C_{\tau}} T(Q) e_{k}(Q),
$$

of the Sugawara operator define a representation of a central extension of the KricheverNovikov vector field algebra given by the geometric cocycle

$$
\chi(e, f)=\frac{\mathrm{c} \cdot \operatorname{dim} \mathfrak{g}}{(\mathrm{c}+\mathrm{k})} \cdot \frac{1}{24 \pi \mathrm{i}} \int_{C_{\tau}}\left(\frac{1}{2}\left(e^{\prime \prime \prime} f-e f^{\prime \prime \prime}\right)-R \cdot\left(e^{\prime} f-e f^{\prime}\right)\right) d z,
$$


with a suitable meromorphic projective connection $R$ with poles only at $P_{ \pm}$and $\operatorname{ord}_{P_{+}}(R) \geq-1$ and $\operatorname{ord}_{P_{-}}(R) \geq-2$.

Remark. The expressions $\psi_{k l}$ in (3-11) define just a coboundary (in the sense of Lie algebra cohomology). Recall a 2 -cocycle $\psi$ is a coboundary of the Lie algebra $\mathcal{L}$ if there is a linear form $\Phi: \mathcal{L} \rightarrow \mathbb{C}$ such that $\psi(e, f)=\Phi([e, f])$. We define $\Phi$ by $\Phi\left(e_{s}\right):=$ $\sum_{v} \sum_{n=0}^{v+1} l_{s}^{n v} \gamma_{n v}$ (this is a finite sum) and calculate $\Phi\left(\left[e_{k}, e_{l}\right]\right)=\Phi\left(\sum_{s} C_{k, l}^{s} e_{s}\right)=\psi_{k l}$. Again a different normal ordering would result in a different range of summation in the definition of $\Phi$ above. By this we proved again, without using the result of Krichever and Novikov on the local cocycles, that different normal ordering would not change the cohomology class of the central extension.

\section{The Proof of Proposition 3.1 And Proposition 3.3}

In the definition of the $L_{k}$ formal infinite sums of operators are involved. To take care about the well-definedness we use cut-off functions as has been done by Kac and Raina in [8]. Let $\psi$ be the function on $\mathbb{R}$ given as

$$
\psi(x)=1 \quad \text { if }|x| \leq 1 \quad \text { and } \quad \psi(x)=0 \quad \text { if }|x|>1 .
$$

For $\epsilon \in \mathbb{R}$ we define

$$
L_{k}(\epsilon)=\frac{1}{2} \sum_{n, m} \sum_{i}: u_{i}(n) u^{i}(m): l_{k}^{n m} \psi(\epsilon n) .
$$

We fix $k$. For every $n$ there are only finitely many $m$ such that $l_{k}^{n m} \neq 0$. Hence for $\epsilon>0$ the sum consists only of finitely many summands. If $v \in V$ then by the normal ordering prescription only finitely many operators $l_{k}^{n m}: u_{i}(n) u^{i}(m)$ : will operate non-trivially on $v$. Hence if we choose $\epsilon>0$ small enough we get $L_{k}(\epsilon) v=L_{k} v$. This we will mean if we write $\lim _{\epsilon \rightarrow 0} L_{k}(\epsilon)=L_{k}$.

If we drop the normal ordering symbols in (4-2) we obtain an expression $\widetilde{L}_{k}(\epsilon)$ which is well-defined as long as $\epsilon \neq 0$. For every pair $(n, m)$ which is not in normal order we take up the commutator $\sum_{i}\left[u_{i}(n), u_{i}(m)\right]$ which is a scalar by Lemma $3.1(3)$, hence $L_{k}(\epsilon)=\widetilde{L}_{k}(\epsilon)+\alpha \cdot t$, where $\alpha$ is a scalar as long as $\epsilon \neq 0$. In particular, if we calculate commutators we can forget about the normal ordering as long as we stay with $\epsilon \neq 0$.

Proof of Proposition 3.1. Per definition we have

$$
\begin{aligned}
R_{\epsilon}:=2\left[\widetilde{L}_{k}(\epsilon), x(r)\right] & =\sum_{n, m} \sum_{i}\left[u_{i}(n) u^{i}(m), x(r)\right] l_{k}^{n m} \psi(\epsilon n) \\
& =\sum_{n, m} \sum_{i}\left(u_{i}(n)\left[u^{i}(m), x(r)\right]+\left[u_{i}(n), x(r)\right] u^{i}(m)\right) l_{k}^{n m} \psi(\epsilon n),
\end{aligned}
$$


after expanding the commutator and reordering the elements again. Each commutator can now be written like

$$
\left[u^{i}(m), x(r)\right]=\left[u^{i}, x\right]\left(A_{m} A_{r}\right)-\left(u^{i} \mid x\right) \gamma_{m r} \cdot \mathrm{c}
$$

(note that $t . v=\mathrm{c} \cdot v$ ). Hence we obtain $R_{\epsilon}=A_{\epsilon}+B_{\epsilon}-\left(C_{\epsilon}+D_{\epsilon}\right)$ where

$$
\begin{aligned}
& A_{\epsilon}=\sum_{n, m} \sum_{i} u_{i}(n)\left[u^{i}, x\right]\left(A_{m} A_{r}\right) l_{k}^{n m} \psi(\epsilon n), B_{\epsilon}=\sum_{n, m} \sum_{i}\left[u_{i}, x\right]\left(A_{n} A_{r}\right) u^{i}(m) l_{k}^{n m} \psi(\epsilon n), \\
& C_{\epsilon}=\sum_{n, m} \sum_{i} u_{i}(n)\left(u^{i} \mid x\right) \gamma_{m r} l_{k}^{n m} \mathrm{c} \psi(\epsilon n), \quad D_{\epsilon}=\sum_{n, m} \sum_{i}\left(u_{i} \mid x\right) u^{i}(m) \gamma_{n r} l_{k}^{n m} \mathrm{c} \psi(\epsilon n) .
\end{aligned}
$$

Now using $\sum_{i} u_{i} \otimes A_{n}\left(u^{i} \mid x\right)=\left(\sum_{i}\left(u^{i} \mid x\right) u_{i}\right) \otimes A_{n}=x \otimes A_{n}=x(n)$, we obtain $C_{\epsilon}=\sum_{n, m} x(n) \gamma_{m r} l_{k}^{n m} \mathrm{c} \psi(\epsilon n)$, and $D_{\epsilon}=\sum_{n, m} x(m) \gamma_{n r} l_{k}^{n m} \mathrm{c} \psi(\epsilon n)$. For fixed $r$ and $k$ only finitely many terms occur. Hence for $\epsilon=0$ we obtain

$$
\lim _{\epsilon \rightarrow 0}\left(C_{\epsilon}+D_{\epsilon}\right)=2 \mathrm{c} \cdot \sum_{n}\left(\sum_{m} l_{k}^{n m} \gamma_{m r}\right) x(n)=2 \mathrm{c} \sum_{n} K_{r, k}^{n} x(n) .
$$

Here we used (3-7). The sums $A_{\epsilon}$ and $B_{\epsilon}$ for $\epsilon \rightarrow 0$ do not make sense separately. We can make sense out of them if we change to normal ordering. For this we have to resolve $A_{m} A_{r}=\sum_{s} \alpha_{m r}^{s} A_{s}$ with $\alpha_{m r}^{s}=\frac{1}{2 \pi \mathrm{i}} \int_{C_{\tau}} A_{m} A_{r} \omega^{s}$. We obtain

$$
A_{\epsilon}=\sum_{n, m, s} \sum_{i} u_{i}(n)\left[u^{i}, x\right](s) \alpha_{m r}^{s} l_{k}^{n m} \psi(\epsilon n), \quad B_{\epsilon}=\sum_{n, m, s} \sum_{i}\left[u_{i}, x\right](s) u^{i}(m) \alpha_{n r}^{s} l_{k}^{n m} \psi(\epsilon n) .
$$

For the elements which are not in normal order we have to pick up a commutator. We write $A_{\epsilon}=A_{\epsilon}^{(1)}+A_{\epsilon}^{(2)}$ and $B_{\epsilon}=B_{\epsilon}^{(1)}+B_{\epsilon}^{(2)}$ where $A_{\epsilon}^{(1)}$ resp. $B_{\epsilon}^{(1)}$ are the expressions above just with the normal ordering columns. We can write the commutator

$$
\left[u_{i}(n),\left[u^{i}, x\right](s)\right]=\left[u_{i},\left[u^{i}, x\right]\right]\left(A_{n} A_{s}\right)-\gamma_{n s}\left(u_{i} \mid\left[u^{i}, x\right]\right) \mathrm{c} .
$$

If we sum over $i$ the second term will vanish because $\left(u_{i} \mid\left[u^{i}, x\right]\right)=\left(\left[u_{i}, u^{i}\right] \mid x\right)$ and Lemma 3.1(2). Lemma 3.1(4) gives for the first term $2 \mathrm{k} \cdot x\left(A_{n} A_{s}\right)=2 \mathrm{k} \sum_{v} \alpha_{n s}^{v} x(v)$. Applying the same to $B_{\epsilon}^{(2)}$ we get

$$
A_{\epsilon}^{(2)}+B_{\epsilon}^{(2)}=2 \mathrm{k} \sum_{v}\left(\sum_{s, m} \sum_{n>s} \alpha_{n s}^{v} \alpha_{m r}^{s} l_{k}^{n m} \psi(\epsilon n)-\sum_{n, m} \sum_{s>m} \alpha_{s m}^{v} \alpha_{n r}^{s} l_{k}^{n m} \psi(\epsilon n)\right) x(v) .
$$

Note that neither sum alone will make sense if we put $\epsilon=0$. Before we continue to deal with (4-4) we first show that $A_{0}^{(1)}+B_{0}^{(1)}$ will vanish. First we change the variables in the summation for $B_{\epsilon}^{(1)}$ in the way $s \rightarrow n \rightarrow m \rightarrow s$. By the normal ordering $A_{0}^{(1)}$ and $B_{0}^{(1)}$ are well-defined operators in the sense that applied to a fixed $v \in V$ only finitely many summands will act nontrivially. Hence we can forget about the $\psi$-factor. 
Lemma 4.1. For $F_{r, k}^{s n}:=\sum_{m} \alpha_{m r}^{s} l_{k}^{n m}=\frac{1}{2 \pi \mathrm{i}} \int_{C_{\tau}} A_{r}(Q) \omega^{s}(Q) \omega^{n}(Q) e_{k}(Q)$ we have $F_{r, k}^{s n}=F_{r, k}^{n s}$.

Proof.

$$
\begin{aligned}
& F_{r, k}^{s n}=\sum_{m} \frac{1}{(2 \pi \mathrm{i})^{2}} \iint_{C_{\tau} C_{\tau^{\prime}}} A_{m}(Q) A_{r}(Q) \omega^{s}(Q) \omega^{n}\left(Q^{\prime}\right) \omega^{m}\left(Q^{\prime}\right) e_{k}\left(Q^{\prime}\right) \\
= & \frac{1}{(2 \pi \mathrm{i})^{2}} \iint_{C_{\tau} C_{\tau^{\prime}}} A_{r}(Q) \omega^{s}(Q) \omega^{n}\left(Q^{\prime}\right) e_{k}\left(Q^{\prime}\right) \Delta\left(Q, Q^{\prime}\right)=\frac{1}{2 \pi \mathrm{i}} \int_{C_{\tau}} A_{r}(Q) \omega^{s}(Q) \omega^{n}(Q) e_{k}(Q) .
\end{aligned}
$$

This is obviously symmetric in $n$ and $s$.

Now $A_{0}^{(1)}+B_{0}^{(1)}=\sum_{n, s} \sum_{i}\left(: u_{i}(n)\left[u^{i}, x\right](s)+\left[u_{i}, x\right](n) u^{i}(s):\right) F_{r, k}^{s n}$.

Lemma 4.2. $\quad \sum_{i}\left(: u_{i}(n)\left[u^{i}, x\right](s)+\left[u_{i}, x\right](n) u^{i}(s):\right)=0$.

Proof. We calculate $\sum_{i} u_{i}(n)\left[u^{i}, x\right](s)=\sum_{i} u_{i}(n) \sum_{j}\left(\left[u^{i}, x\right] \mid u_{j}\right) u^{j}(s)=$ $-\sum_{i, j} u_{i}(n)\left(u^{i} \mid\left[u_{j}, x\right]\right) u^{j}(s)=-\sum_{j}\left[u_{j}, x\right](n) u^{j}(s)$.

Hence the $A_{0}^{(1)}+B_{0}^{(1)}=0$.

We now take up (4-4) again.

Claim. The expression inside the $v$-summation is for $\lim _{\epsilon \rightarrow 0}$ equal to the $\lim _{\epsilon \rightarrow 0}$ of

$$
E_{\epsilon}^{(N)}:=\sum_{m, s} \sum_{n>N} \alpha_{n s}^{v} \alpha_{m r}^{s} l_{k}^{n m} \psi(\epsilon n)-\sum_{n, m} \sum_{s>N} \alpha_{s m}^{v} \alpha_{n r}^{s} l_{k}^{n m} \psi(\epsilon n)
$$

where $N$ is an arbitrary integer.

Proof. If we calculate the difference we obtain ${ }^{1}$

$$
\sum_{m, s} \sum_{n=s+1}^{N} \alpha_{n s}^{v} \alpha_{m r}^{s} l_{k}^{n m} \psi(\epsilon n)-\sum_{n, m} \sum_{s=m+1}^{N} \alpha_{s m}^{v} \alpha_{n r}^{s} l_{k}^{n m} \psi(\epsilon n) .
$$

Note that due to the almost-grading in each sum for fixed $v, k, r$ only finitely many terms are involved. Hence we can forget about $\psi(\epsilon n)$ and change variables $(s \rightarrow n \rightarrow m \rightarrow s)$ in the second sum. Applying Lemma 4.1 we see that the difference will vanish.

\footnotetext{
${ }^{1}$ If the upper bound is smaller than the lower bound, we mean that one has to switch the summation range and the sign of the expression. This will be understood in all the summations which will follow.
} 
This proof shows also that our result will not depend on the normal ordering chosen. Again the difference will consist of finitely many terms which will cancel.

We will study

$$
E_{\epsilon}^{(0)}=\sum_{s} \sum_{n>0} \alpha_{n s}^{v} F_{r, k}^{s n} \psi(\epsilon n)-\sum_{n} \sum_{s>0} \alpha_{n r}^{s} F_{s, k}^{v n} \psi(\epsilon n)
$$

We replace the second summation range as follows

$(n, s>0)=(s, n>0)+(n>0, s \leq 0)-(s>0, n \leq 0)$ and obtain

$$
E_{\epsilon}^{(0)}=\sum_{n>0} \sum_{s}\left(\alpha_{n s}^{v} F_{r, k}^{s n}-\alpha_{n r}^{s} F_{s, k}^{v n}\right) \psi(\epsilon n)+\left(\sum_{\substack{n>0 \\ s \leq 0}}-\sum_{\substack{s>0 \\ s \leq 0}}\right) \alpha_{n r}^{s} F_{s, k}^{v n} \psi(\epsilon n) .
$$

After summation over $s$ in the first sum and using the "delta distribution" we see that it will vanish. Using the integral representation of $F_{s, k}^{v n}$ (Lemma 4.1) and of $\alpha_{n r}^{s}(2-14)$ the second part can be rewritten as

$$
\frac{1}{(2 \pi \mathrm{i})^{2}} \iint_{C_{\tau} C_{\tau^{\prime}}} A_{r}\left(Q^{\prime}\right) e_{k}(Q) \omega^{v}(Q)\left(\sum_{\substack{n>0 \\ s \leq 0}}-\sum_{\substack{s>0 \\ n \leq 0}}\right) A_{n}\left(Q^{\prime}\right) A_{s}(Q) \omega^{s}\left(Q^{\prime}\right) \omega^{n}(Q) \psi(\epsilon n) .
$$

Now we use

Lemma 4.3. (Bonora et al. [1]) For every $N$ we have

$$
\left(\sum_{n>N} \sum_{s \leq N}-\sum_{s>N} \sum_{n \leq N}\right) A_{n}\left(Q^{\prime}\right) A_{s}(Q) \omega^{s}\left(Q^{\prime}\right) \omega^{n}(Q)=d^{\prime} \Delta\left(Q^{\prime}, Q\right) .
$$

Here $d^{\prime}$ means differentiation with respect to the variable $Q^{\prime}$.

For completeness we will supply a proof of it below. Applying Lemma 4.3 to our situation we obtain

$$
\begin{aligned}
& E_{0}^{(0)}=\frac{1}{(2 \pi \mathrm{i})^{2}} \iint_{C_{\tau} C_{\tau^{\prime}}} A_{r}\left(Q^{\prime}\right) e_{k}(Q) \omega^{v}(Q) d^{\prime} \Delta\left(Q^{\prime}, Q\right) \\
= & -\frac{1}{(2 \pi \mathrm{i})^{2}} \iint_{C_{\tau}} d_{C_{\tau^{\prime}}} d^{\prime} A_{r}\left(Q^{\prime}\right) e_{k}(Q) \omega^{v}(Q) \Delta\left(Q^{\prime}, Q\right)=-\frac{1}{2 \pi \mathrm{i}} \int_{C_{\tau}} d A_{r}(Q) e_{k}(Q) \omega^{v}(Q)=-K_{r, k}^{v} .
\end{aligned}
$$

If we collect all the non-vanishing parts we just obtain the claim of Proposition 3.1. 
Proof of Lemma 4.3. First we proof the following relation

Lemma 4.4.

$$
\gamma_{r k}=\left(\sum_{n>0} \sum_{s \leq 0}-\sum_{s>0} \sum_{n \leq 0}\right) \alpha_{r n}^{s} \alpha_{k s}^{n}
$$

Proof. It is the idea of Bonara and collaborators to use representations via semi-infinite forms. Here we take forms of weight 0 . Take $\Phi=A_{1} \wedge A_{2} \wedge \ldots$ the vacuum vector of weight 0 and level 1. The element $A_{i} \in \mathcal{A}$ operates with Leibnitz rule as

$$
A_{i} . \Phi=\left(A_{i} \cdot A_{1}\right) \wedge A_{2} \wedge \ldots+A_{1} \wedge\left(A_{i} \cdot A_{2}\right) \wedge A_{3} \ldots+\ldots
$$

As long as $|i|$ is big enough this makes perfect sense. For some critical strip of indices (e.g. in particular for $A_{0}=1$ ) the action has to be modified $^{2}$ and we obtain only a representation of a centrally extended algebra $\widehat{\mathcal{A}}^{\prime}$ where the defining cocycle is a local one [10]. Because $\mathcal{A}$ is an abelian Lie algebra two different cocycles are never cohomologous. Similar to the case of the vector field algebra it can be proven that there is only one (up to multiplication by a scalar $d$ ) local cocycle for the algebra $\mathcal{A}$ ([10], [9]). Hence $\left[\widehat{A}_{r}, \widehat{A}_{k}\right] . \Phi=d \gamma\left(A_{r}, A_{k}\right) \Phi=d \gamma_{r k} \Phi$. If $r$ and $k$ are outside of the critical strip of the indices the action of $\widehat{A}_{r}$, resp. $\widehat{A}_{k}$ coincides with the action of the corresponding element $A$ given by (4-12). Inside the critical strip at least $\left[\widehat{A}_{r}, \widehat{A}_{k}\right] . \Phi$ can be calculated as follows (e.g. see [16, p.137]) for details). One has to take only in account the ways the element $A_{s}$ inside $\Phi$ will reproduce itself. First $A_{k} \cdot A_{s}=\sum_{n} \alpha_{k s}^{n} A_{n}$. This term will only occur if $s \geq 1$ and only the terms with $n<1$ will not be annihilated by neighbouring elements. To bring it back to $A_{s}$ by operation of $A_{r}$ we obtain $\sum_{s>0} \sum_{n \leq 0} \alpha_{r n}^{s} \alpha_{k s}^{n}$. Applying the same to $-A_{k} \cdot A_{r}$, and changing the variables we obtain

$$
\left[\widehat{A}_{r}, \widehat{A}_{k}\right] . \Phi=-\left(\sum_{n>0} \sum_{s \leq 0}-\sum_{s>0} \sum_{n \leq 0}\right) \alpha_{r n}^{s} \alpha_{k s}^{n} \Phi=d \gamma_{r k} \Phi
$$

To determine the constant we calculate this expression for $r=i$ and $k=-i$ if $i \gg 0$. Note that $A_{i} \Phi=0$, hence $\left[A_{i}, A_{-i}\right] \Phi=A_{i}\left(A_{-i} \Phi\right)$ and that $A_{k} \cdot A_{s}=A_{k+s}+A_{j}$-terms with indices of $j$ bigger as $k+s$. Hence we pick up for every $s$ the factor 1 as long as $s-i \leq 0$. Now $s \geq 1$ and we obtain $\left[A_{i}, A_{-i}\right] \Phi=i \cdot \Phi=d \cdot \gamma\left(A_{i}, A_{-i}\right) \Phi$. But by calculating residues we get $\gamma\left(A_{i}, A_{-i}\right)=-i$ and hence the claim.

Note that Bonora et al. also indicate a proof by direct calculation.

Now

$$
d A_{k}(Q)=\sum_{r} \beta_{k r} \omega^{r}(Q), \quad \beta_{k r}=\frac{1}{2 \pi \mathrm{i}} \int_{C_{\tau}} d A_{k}(Q) A_{r}(Q)=\gamma_{r k},
$$

\footnotetext{
${ }^{2}$ In physicists' language, it has to be regularized.
} 
and hence using Lemma 4.4

$$
d^{\prime} \Delta\left(Q^{\prime}, Q\right)=\sum_{k} d^{\prime} A_{k}\left(Q^{\prime}\right) \omega^{k}(Q)=\sum_{k, r}\left(\sum_{\substack{n>0 \\ s \leq 0}}-\sum_{\substack{s>0 \\ n \leq 0}}\right) \alpha_{r n}^{s} \alpha_{k s}^{n} \omega^{k}(Q) \omega^{r}\left(Q^{\prime}\right)
$$

Now using that $A_{i} \omega^{j}=\sum_{r} \alpha_{i r}^{j} \omega^{r}$ we obtain the result for $N=0$. To get it for general $N$, we just compare the summation ranges with the $N=0$ range. We obtain as difference $\left(\sum_{s=1}^{N} \sum_{n}-\sum_{n=1}^{N} \sum_{s}\right) \alpha_{r n}^{s} \alpha_{k s}^{n} \omega^{k}(Q) \omega^{r}\left(Q^{\prime}\right)$. Now each of the partial sums has only finitely many terms. Hence we can do the summation separately. But doing this for the summation over $n$ in the first sum (after writing the coefficients as integrals and using the "delta distribution") and over $s$ in the second sum we obtain the same value and they will cancel.

Proof of Proposition 3.3. Again we write for $\epsilon \neq 0$.

$$
\left[L_{k}(\epsilon), L_{l}\right]=\frac{1}{2} \sum_{n, m} \sum_{i} l_{k}^{n m}\left[: u_{i}(n) u^{i}(m):, L_{l}\right] \psi(\epsilon n) .
$$

As explained at the beginning of this section we can ignore the normal ordering inside the above commutators and rewrite (4-16) as

$$
\frac{1}{2} \sum_{n, m} \sum_{i} l_{k}^{n m}\left(u_{i}(n)\left[u^{i}(m), L_{l}\right]+\left[u_{i}(n), L_{l}\right] u^{i}(m)\right) \psi(\epsilon n) .
$$

We use Prop. 3.1 to evaluate the commutators and obtain

$$
\frac{1}{2}(\mathrm{c}+\mathrm{k}) \sum_{n, m, v} \sum_{i} l_{k}^{n m}\left(K_{m, l}^{v} u_{i}(n) u^{i}(v)+K_{n, l}^{v} u_{i}(v) u^{i}(m)\right) \psi(\epsilon n) .
$$

To make things separately well-defined for $\epsilon=0$ we have to rewrite everything again in normal order and pick up commutators for indices which are not in normal order. After evaluating this commutators using Lemma 3.1(3) we can write the result as sum $A_{\epsilon}+B_{\epsilon}+C_{\epsilon}+D_{\epsilon}$ where they are defined as

$$
\begin{aligned}
& A_{\epsilon}=\frac{1}{2}(\mathrm{c}+\mathrm{k}) \sum_{n, m, v} \sum_{i} l_{k}^{n m} K_{m, l}^{v}: u_{i}(n) u^{i}(v): \psi(\epsilon n), \\
& B_{\epsilon}=\frac{1}{2}(\mathrm{c}+\mathrm{k}) \sum_{n, m, v} \sum_{i} l_{k}^{n m} K_{n, l}^{v}: u_{i}(v) u^{i}(m): \psi(\epsilon n), \\
& C_{\epsilon}=-\frac{1}{2} \mathrm{c}(\mathrm{c}+\mathrm{k}) \operatorname{dim} \mathfrak{g} \sum_{v, m} \sum_{n>v} l_{k}^{n m} K_{m, l}^{v} \gamma_{n v} \psi(\epsilon n), \\
& D_{\epsilon}=-\frac{1}{2} \mathrm{c}(\mathrm{c}+\mathrm{k}) \operatorname{dim} \mathfrak{g} \sum_{n, m} \sum_{v>m} l_{k}^{n m} K_{n, l}^{v} \gamma_{v m} \psi(\epsilon n) .
\end{aligned}
$$


By considering the range where the coefficients $l_{k}^{n m}$ and $K_{m, l}^{v}$ could be nonzero and taking the normal ordering into account we see that $A_{0}$ and $B_{0}$ are again well-defined operators and we can ignore the $\psi(\epsilon n)$ factor. Renaming the variables in $B_{0}$ in the way $(v \rightarrow n \rightarrow m \rightarrow v)$ we obtain

$$
A_{0}+B_{0}=\frac{1}{2}(\mathrm{c}+\mathrm{k}) \sum_{n, m, v} \sum_{i}\left(l_{k}^{n m} K_{m, l}^{v}+l_{k}^{m v} K_{m, l}^{n}\right): u_{i}(n) u^{i}(v):
$$

The structure constants of the vector field algebra $\mathcal{L}$ can be calculated as

$$
C_{k l}^{s}=\frac{1}{2 \pi \mathrm{i}} \int_{C_{\tau}}\left(\left[e_{k}, e_{l}\right]\right) \cdot \Omega^{s}
$$

\section{Lemma 4.5.}

$$
\sum_{m}\left(l_{k}^{n m} K_{m, l}^{v}+l_{k}^{m v} K_{m, l}^{n}\right)=-\frac{1}{2 \pi \mathrm{i}} \int_{C_{\tau}}\left[e_{k}, e_{l}\right] \cdot \omega^{n} \omega^{v}=-\sum_{s} C_{k l}^{s} l_{s}^{n v} .
$$

Proof. We can write the right hand side as

$$
-\sum_{s} \frac{1}{(2 \pi \mathrm{i})^{2}} \iint_{C_{\tau}}\left[e_{\tau_{\tau^{\prime}}}, e_{l}\right](Q) \cdot \Omega^{s}(Q) \omega^{n}\left(Q^{\prime}\right) \omega^{v}\left(Q^{\prime}\right) e_{s}\left(Q^{\prime}\right)
$$

After summation over $s$ we obtain the "delta distribution" for the pair $(-1,2)$, integrate over $Q^{\prime}$ and obtain $-\frac{1}{2 \pi \mathrm{i}} \int_{C_{\tau}}\left[e_{k}, e_{l}\right](Q) \omega^{n}(Q) \omega^{v}(Q)$, the expression in the middle. On the left hand side we obtain for the first sum

$$
\sum_{m} l_{k}^{n m} K_{m, l}^{v}=\sum_{m} \frac{1}{(2 \pi \mathrm{i})^{2}} \iint_{C_{\tau} C_{\tau^{\prime}}} \omega^{n}(Q) \omega^{m}(Q) e_{k}(Q) d^{\prime} A_{m}\left(Q^{\prime}\right) e_{l}\left(Q^{\prime}\right) \omega^{v}\left(Q^{\prime}\right)
$$

Now applying $\sum_{m} d^{\prime} A_{m}\left(Q^{\prime}\right) \omega^{m}(Q)=d^{\prime} \Delta\left(Q^{\prime}, Q\right)$ we obtain after integration over $Q^{\prime}$ $-\frac{1}{2 \pi \mathrm{i}} \int_{C_{\tau}} \omega^{n}(Q) e_{k}(Q) d\left(e_{l}(Q) \omega^{v}(Q)\right)$. For the second sum we obtain

$-\frac{1}{2 \pi \mathrm{i}} \int_{C_{\tau}} \omega^{v}(Q) e_{k}(Q) d\left(e_{l}(Q) \omega^{n}(Q)\right)=\frac{1}{2 \pi \mathrm{i}} \int_{C_{\tau}} d\left(\omega^{v}(Q) e_{k}(Q)\right) e_{l}(Q) \omega^{n}(Q)$. Together

$$
-\frac{1}{2 \pi \mathrm{i}} \int_{C_{\tau}}\left(\omega^{n}(Q) e_{k}(Q) d\left(e_{l}(Q) \omega^{v}(Q)\right)-\omega^{n}(Q) e_{l}(Q) d\left(e_{k}(Q) \omega^{v}(Q)\right)\right) \text {. }
$$

If we represent each form by its local representing function we obtain for the integrand

$$
\omega^{n}(z) \omega^{v}(z)\left(e_{k}(z) \frac{d e_{l}}{d z}(z)-e_{l}(z) \frac{d e_{k}}{d z}(z)\right)=\omega^{n}(z) \omega^{v}(z)\left[e_{k}, e_{l}\right](z)
$$


Hence the claim.

Now

$$
A_{0}+B_{0}=-\frac{1}{2}(\mathrm{c}+\mathrm{k}) \sum_{s} \sum_{n, v} \sum_{i} C_{k l}^{s} l_{s}^{n v}: u_{i}(n) u^{i}(v):=-(\mathrm{c}+\mathrm{k}) \sum_{s} C_{k l}^{s} L_{s} .
$$

It remains to study $\alpha(k, l):=\lim _{\epsilon \rightarrow 0}\left(C_{\epsilon}+D_{\epsilon}\right)$. Because $L_{k}$ and $L_{l}$ are well-defined operators inside $g l(V)$ the scalar $\alpha(k, l)$ is well-defined. Indeed, using the Jacobi identity inside $g l(V)$ and the fact that the $C_{k l}^{s}$ fulfil also the Jacobi identity (as they are the structure constants of $\mathcal{L}$ ) we obtain that it defines a 2-cocycle. By studying the order of the forms involved in defining $l_{k}^{n m}, K_{m, l}^{v}$ and $\gamma_{n v}$ at the points $P_{+}$and $P_{-}$and calculating residues to evaluate the integral we see that for generic $n, m, v$ values

$$
\begin{gathered}
K_{m, l}^{v} \neq 0 \Longrightarrow-3 g \leq-v+l+m \leq 0 \\
l_{k}^{n m} \neq 0 \Longrightarrow-g \leq k-(n+m) \leq 0, \quad \gamma_{n v} \neq 0 \Longrightarrow-2 g \leq n+v \leq 0 .
\end{gathered}
$$

Adding them up we obtain $\alpha(k, l) \neq 0 \Longrightarrow-6 g \leq k+l \leq 0$. For $g \neq 0$ and $n=-g$ and $v=-g-1$ (or vice versa) $\gamma_{-g,-g-1}$ will be non-zero. But in this case the other coefficients will again yield the same bound. In particular the cocycle is local. It can be given as

$$
\alpha(k, l)=-\frac{1}{2} \mathrm{c}(\mathrm{c}+\mathrm{k}) \operatorname{dim} \mathfrak{g} \sum_{n}\left(\sum_{m, s} \sum_{v<n} l_{k}^{n m} l_{l}^{v s} \gamma_{s m} \gamma_{n v}+\sum_{m, s} \sum_{v>m} l_{k}^{n m} l_{l}^{v s} \gamma_{s n} \gamma_{v m}\right) .
$$

In the rest of this section we want to find a nicer representation of the cocycle. We do not want to take the overall factor $(-1 / 2) \mathfrak{c}(\mathfrak{c}+k) \operatorname{dim} \mathfrak{g}$ through all the calculation. So we just ignore it in the calculation and keep it in mind. Let $N$ be a fixed integer. We start again from $C_{\epsilon}$ and $D_{\epsilon}$. We define $E_{\epsilon}:=\sum_{v, m} \sum_{n>N} l_{k}^{n m} K_{m, l}^{v} \gamma_{n v} \psi(\epsilon n)$, and $F_{\epsilon}:=\sum_{n, m} \sum_{v>N} l_{k}^{n m} K_{n, l}^{v} \gamma_{v m} \psi(\epsilon n)$. In $C_{\epsilon}-E_{\epsilon}$ and $D_{\epsilon}-F_{\epsilon}$ only finitely many terms will occur. Hence we can put $\epsilon=0$ and forget about $\psi(\epsilon n)$. If we rename in $D_{\epsilon}-F_{\epsilon}$ the variables $(v \rightarrow n \rightarrow m \rightarrow v)$ we obtain the following expression

$$
\begin{aligned}
\lim _{\epsilon \rightarrow 0}\left(\left(C_{\epsilon}-E_{\epsilon}\right)+\left(D_{\epsilon}-F_{\epsilon}\right)\right) & =\sum_{v} \sum_{n=v+1}^{N}\left(\sum_{m} l_{k}^{n m} K_{m, l}^{v}+l_{k}^{m v} K_{m l}^{n}\right) \gamma_{n v} \\
& =-\sum_{v} \sum_{n=v+1}^{N} \sum_{s} C_{k l}^{s} l_{s}^{n v} \gamma_{n v},
\end{aligned}
$$

by applying Lemma 4.5. We obtain that contrary to the case considered in the proof of Prop. 3.1 this will not vanish. In particular this is the reason that the value of the cocycle will depend on the normal ordering chosen. 
Now we are examining $E_{\epsilon}+F_{\epsilon}$. We decompose the summation range for $F_{\epsilon}$ as $(n, v>N)=(v, n>N)+(v>N, n \leq N)-(n>N, v \leq N)$ and call the first sum $F_{\epsilon}^{(1)}$ and the two others together $F_{\epsilon}^{(2)}$. First we obtain by using $\gamma_{v m}=-\gamma_{m v}$ and $K_{v, k}^{n}=\sum_{m} l_{k}^{n m} \gamma_{m v}$

$$
E_{\epsilon}+F_{\epsilon}^{(1)}=\sum_{v} \sum_{n>N}\left(\left(\sum_{m} l_{k}^{n m} K_{m, l}^{v}\right) \gamma_{n v}-K_{n, l}^{v} K_{v, k}^{n}\right) \psi(\epsilon n) .
$$

Because $F_{\epsilon}^{(2)}$ will be well-defined for $\epsilon=0$ (see below) we can ignore here $\psi(\epsilon n)$ as long as we not break it into the two partial sums. Using the integral representation of the coefficients and performing the $m$-summation we get a "delta distribution" (as we did it several times) and obtain

$$
\begin{aligned}
\left(\sum_{m} l_{k}^{n m} K_{m, l}^{v}\right) \gamma_{n v} & =-\frac{1}{(2 \pi \mathrm{i})^{2}} \iint_{C_{\tau}} w_{\tau^{\prime}}^{v}(Q) d^{\prime} A_{n}\left(Q^{\prime}\right) e_{l}(Q) d\left(e_{k}(Q) \omega^{n}(Q)\right) A_{v}\left(Q^{\prime}\right) \\
K_{n, l}^{v} K_{v, k}^{n} & =\frac{1}{(2 \pi \mathrm{i})^{2}} \iint_{C_{\tau}} w_{C_{\tau^{\prime}}} w^{v}\left(Q^{\prime}\right) d^{\prime} A_{n}\left(Q^{\prime}\right) e_{l}\left(Q^{\prime}\right) d\left(e_{k}(Q) \omega^{n}(Q)\right) A_{v}(Q)
\end{aligned}
$$

Summation over $v$ and integration over $Q^{\prime}$ shows that $E_{\epsilon}+F_{\epsilon}^{(1)}=0$.

It remains to look at

$$
F_{\epsilon}^{(2)}=\left(\sum_{n>N} \sum_{v \leq N}-\sum_{n \leq N} \sum_{v>N}\right) K_{v, k}^{n} K_{n, l}^{v} \psi(\epsilon n)
$$

By checking the structure constants we see that every partial sum consists only of finitely many terms. Hence we can set again $\epsilon=0$. For $N=0$ we collect the surviving terms (4-22) and (4-23) and obtain the result of the structure of the cocycle (3-11) as claimed in Prop. 3.3. If we choose a different normal ordering we would just obtain a different summation prescription in (4-22). The expression (4-23) would be the same. It remains to show the form (3-12) of the cocycle for the special basis elements. We look first at $\widehat{\chi}_{k,-k}$. Now $K_{v, k}^{n} \neq 0$ implies $k \leq n-v$ and $K_{n,-k}^{v} \neq 0$ implies $-k \leq v-n$, hence $v=n-k$. And as value we obtain in this case $(n-k)$ resp. $n$. Assume $k \geq 0$ then only the first sum will survive. It will be the finite sum

$$
\sum_{n=1}^{k}(n-k) n=-\frac{1}{6}\left(k^{3}-k\right)
$$

In the expression for $\psi_{k,-k}$ we obtain that $C_{k,-k}^{s} l_{s}^{n v} \gamma_{n v} \neq 0$ only if $s \geq 0, s \leq n+v$ and $n+v \leq 0$. This implies that we obtain non-zero terms only for $s=0$ and $n=-v$. 
But if we look at the summation range $\sum_{v} \sum_{n=0}^{v+1}$ we see that $n=-v$ imposes that either $v=0$ (with vanishing coefficient) or that the summation range is empty. Hence $\psi_{k,-k}=0$. Altogether we get the form (3-12).

For further reference let us note

Corollary 4.1. Let $E$ be an operator of $g l(V), V$ the fixed admissible representation chosen in Section 3, such that there exists a basis element $e_{l}$ of $\mathcal{L}$ with

$$
[E, x(r)]=-(\mathrm{c}+\mathrm{k}) x\left(\nabla_{e_{l}} A_{r}\right)
$$

for every $x \in \mathfrak{g}$ and every $r$, then for every $k$ we have

$$
\left[L_{k}, E\right]=\left[L_{k}, L_{l}\right]
$$

where the latter can be evaluated by (3-10).

Proof. In the proof of Prop. 3.3 we used only the relation $\left[L_{l}, x(r)\right]=-(\mathrm{c}+\mathrm{k}) x \otimes$ $\left(\nabla_{e_{l}} A_{r}\right)$ of Prop. 3.2. Hence if $E$ is an operator obeying the same rules we will obtain $\left[L_{k}(\epsilon), E\right]=\left[L_{k}(\epsilon), L_{l}\right]$ and hence also in the limit $\epsilon \rightarrow 0:\left[L_{k}, E\right]=\left[L_{k}, L_{l}\right]$.

\section{The weight of the Sugawara Representation}

Assume that we have a $\widehat{\mathcal{G}}$-module with a certain highest weight such that $\widehat{\mathcal{L}}$ acts via the corresponding Sugawara representation on it as introduced in Section 5. The goal of this section is to express the weight of the Sugawara representation in terms of the weight of the $\widehat{\mathcal{G}}$-module under consideration. The following result will generalize the result of [20], which was obtained for the Krichever-Novikov algebras of Heisenberg type.

The theory of highest weight modules for Krichever-Novikov algebras of affine type is developed in [19] (see also [20]). Let us outline the class of highest weight modules we are considering here.

Let $\mathfrak{g}$ be again a fixed finite-dimensional simple Lie algebra. We denote by $\mathfrak{h}$ a fixed Cartan subalgebra, by $\mathfrak{n}_{+}$(resp. $\mathfrak{n}_{-}$) its upper (resp. lower) nilpotent algebra, and by $\mathfrak{h}^{*}$ the dual of the Cartan subalgebra. As usual $U(B)$ denotes the universal enveloping algebra of the Lie algebra $B$. Note that $\mathfrak{g}$ is embedded via $x \mapsto x \otimes A_{0}$ into $\mathcal{G}_{0}$ and $\widehat{\mathcal{G}}_{0}$. Hence $\mathfrak{h}, \mathfrak{n}_{+}, \mathfrak{n}_{-}$can be considered as subalgebras of $\widehat{\mathcal{G}}$. 
Let $V$ be an almost-graded $\widehat{\mathcal{G}}$-module. Recall from Section 2 that $\widehat{\mathcal{G}}$ is an almostgraded Lie algebra. Let us recall the definition of an almost-graded module. We have given a $\mathbb{Z}$-degree $\operatorname{deg}$ on $V$. The elements $x$ with $\operatorname{deg} x=n$ are called homogeneous elements of degree $n$. Denote by $V_{n}$ the subspace generated by all homogeneous elements of degree $n$. It is assumed to be finite dimensional ${ }^{3}$ for every $n$ and $V$ is the vector space direct sum $V=\sum_{n \in \mathbb{Z}} V_{n}$. Concerning the action of $\widehat{\mathcal{G}}$ on $V$, there are constants $K, L$ such that for all $n$ and $m$ we have

$$
\widehat{\mathcal{G}}_{m} \cdot V_{n} \subseteq \sum_{h=n+m-K}^{n+m+L} V_{h} .
$$

Because we are heading for the Sugawara representation we will assume that a certain normal ordering :...: has been fixed. We will suppose that $V$ together with the normal ordering satisfy the following conditions:

(1) There exists an element $v \in V$ such that

$$
\widehat{\mathcal{G}}_{+} v=\mathfrak{n}_{+} v=0, \quad \text { and } \quad V=U(\widehat{\mathcal{G}}) v \text {. }
$$

(2) There exists $\mathfrak{c} \in \mathbb{C}$ and $\chi=\left(\chi_{0}, \chi_{-1}, \ldots, \chi_{-g}\right) \in\left(\mathfrak{h}^{*}\right)^{g+1}$, such that for all $m, n \in\{0,-1, \ldots,-g\}$ and all $h, h^{\prime} \in \mathfrak{h}$ we have

$$
h(m) v=\chi_{m}(h) v+\ldots, \quad \text { and } \quad t . v=\mathrm{c} v,
$$

and

$$
: h(m) h^{\prime}(n): v=\chi_{m}(h) \chi_{n}\left(h^{\prime}\right) v+\ldots .
$$

Here and in the following ... denotes terms of lower degree. Recall that $t$ is the basis element of the center. Such a tuple $\chi$ is called the weight, $\mathrm{c}$ is called the central charge, and $v=v_{\chi, c}$ is called the highest weight vector of the module $V$.

(3) For any positive finite root $\alpha$ and corresponding root vector $x_{\alpha}$ we have for all $m=0,-1, \ldots,-g$,

$$
x_{\alpha}(m) v=0 \cdot v+\ldots .
$$

(4) The almost-grading of the $\widehat{\mathcal{G}}$-module $V$ is compatible with the almost-grading of the associative algebra of functions $\mathcal{A}$ in the following sense. For $n, m \leq-g$ and every $v_{m} \in V$ with $\operatorname{deg} v_{m}=m$ we have that in $u(n) v_{m}$ elements of degree $k$ could only occur if in $A_{n} A_{m}$ elements of degree $k$ are occuring. In formulas, if $\left\{v_{k}^{1}, v_{k}^{2}, \ldots, v_{k}^{r}\right\}$ are basises of the spaces $V_{k}$ then

$$
\begin{aligned}
\left\{k \mid u(n) v_{m}=\sum_{k, s} d_{n m}^{k, s}(u) v_{k}^{s},\right. & \left.\exists s: d_{n m}^{k, s}(u) \neq 0\right\} \\
& \subseteq\left\{k \mid A_{n} A_{m}=\sum_{k} \alpha_{n m}^{k} A_{k}, \exists \alpha_{n m}^{k} \neq 0\right\} .
\end{aligned}
$$

\footnotetext{
${ }^{3}$ For certain applications the finite dimensionality of the homogeneous subspaces might be dropped. With respect of this more general conditions our modules are what is called "quasi-finite modules".
} 
(5) In addition we suppose that $\operatorname{deg} v<-g$ and that all elements of degree $\geq \operatorname{deg} v$ are multiples of $v$.

Conditions (4) and (5) will take care that for $n<-g$ the vector $x(n) v$ in its decomposition will contain only elements of degree less than the degree of $v$.

Now we are coming to the Lie algebra $\widehat{\mathcal{L}}$. We call a tuple $\lambda=\left(\lambda_{0}, \lambda_{-1}, \ldots, \lambda_{-3 g}\right) \in$ $\mathbb{C}^{3 g+1}$ a weight of the Lie algebra $\widehat{\mathcal{L}}$. To each weight $\lambda$ we assign the quadratic differential

$$
\Lambda=\sum_{k=-3 g}^{0} \lambda_{k} \Omega^{k}
$$

where the $\Omega^{k}$ are the basis elements introduced in Section 2, see (2-11). This quadratic differential $\Lambda$ will be called a weight of the Lie algebra $\widehat{\mathcal{L}}$ as well. Note that also for the $\widehat{\mathcal{G}}$-modules (resp. for the $\widehat{\mathcal{G}}^{e}$-modules which we are considering in the next section) the weights can be identified with the abelian differentials of first order [19,20].

Let $V$ be an $\widehat{\mathcal{L}}$-module. In analogy with (1)-(5) we impose the following conditions on the module $V$.

(6) There is an element $v \in V$ such that

$$
\widehat{\mathcal{L}}_{+} v=0, \quad \text { and } \quad V=U(\widehat{\mathcal{L}}) v
$$

(7) There exists a weight $\lambda$ such that

$$
E_{m} v=\lambda_{m} v+\ldots
$$

for each $m=0,-1, \ldots,-3 g$. Here we denote by $E_{m}$ the element $\left(e_{m}, 0\right)$ which is the lift of the vector field $e_{m} \in \mathcal{L}$ to the central extension $\widehat{\mathcal{L}}$.

Now let us define more precisely which classes of normal orderings $\Sigma$ we consider in this section. Let $\Sigma^{ \pm}$be a decomposition of the two-dimensional lattice $\{(m, n) \mid m, n \in \mathbb{Z}\}$ into two disjoint parts such that the $\Sigma^{ \pm}$differ from

$$
\Sigma_{0}^{ \pm}=\{(m, n) \mid m \leq n(\text { resp. } m>n), m, n \in \mathbb{Z}\}
$$

only for $-g \leq m, n \leq 0$. According to [10] each decomposition of this kind defines a normal ordering by setting

$$
: x(m) y(n):=\left\{\begin{array}{ll}
x(m) y(n) & ,(m, n) \in \Sigma^{+} \\
y(n) x(m) & ,(m, n) \in \Sigma^{-}
\end{array} .\right.
$$


for each $x, y \in \mathfrak{g}$, and each pair $(m, n)$.

More general normal orderings with the only restriction that they differ from $\Sigma_{0}$ at most for finitely many pairs $(m, n)$ are possible. There is no essential difficulty involved. It is only that the equations in the following would look slightly more complicated.

Warning. For abelian $\mathfrak{g}$ by the decomposition $\Sigma^{ \pm}$the order of the elements inside the normal ordering colons does not play any role, e.g. $: x(n) y(m):=: y(m) x(n):$ For non-abelian $\mathfrak{g}$ this is only true for the elements of the Cartan subalgebra $\mathfrak{h}$.

For further reference let us denote $\Sigma_{c s}^{ \pm}:=\Sigma^{ \pm} \cap\{(m, n) \mid-g \leq m, n \leq 0, m, n \in \mathbb{Z}\}$.

In the rest of this section we would like to connect $\chi$ and $\lambda$ assuming that $V$ is a $\widehat{\mathcal{G}}$-module of highest weight $\chi$ and $\lambda$ is the weight of the corresponding Sugawara representation. Assume that $V$ obeys the Conditions (1)-(5). Let $\chi$ be its weight, $\mathrm{c}$ its central charge and $v$ its highest weight vector. By (5-1) we see that it is an admissible module in the sense of Section 3. Hence we can apply the Sugawara construction. Assume $(c+k) \neq 0$ (which is for positive $c$ always the case), then we obtain by mapping $E_{k}$ to $L_{k}^{*}=\frac{-1}{\mathrm{c}+\mathrm{k}} L_{k}$, the rescaled modes of the Sugawara operator, and mapping the central element of $\widehat{\mathcal{L}}$ to the identity operator on $V$ a representation of $\widehat{\mathcal{L}}$. This is the content of Theorem 3.1.

Lemma 5.1. Independently of the normal ordering chosen for this representation of $\widehat{\mathcal{L}}$ we have

$$
L_{k}^{*} v=0, \quad \text { for all } k>0,
$$

and

$$
L_{k}^{*} v=\lambda_{k} v+\ldots, \quad \text { for } k=0,-1, \ldots,-3 g,
$$

with certain $\lambda_{k} \in \mathbb{C}$.

The elements $L_{k}^{*} v$ for $k<-3 g$ contain in their degree decomposition only elements $w$ with $\operatorname{deg} w<\operatorname{deg} v$.

In particular the Condition (6) and (7) are fulfilled for the subspace $U(\widehat{\mathcal{L}}) v$.

Proof. By definition $L_{k}^{*} v$ is up to a scalar given as

$$
w:=\sum_{n, m} \sum_{i} l_{k}^{m n}: u_{i}(m) u^{i}(n): v
$$

Note that due to the summation over $i$ (see Lemma 3.1(3)) we obtain

$$
\sum_{i} l_{k}^{m n} u_{i}(m) u^{i}(n)=\sum_{i} l_{k}^{m n} u_{i}(m) u^{i}(n)-\operatorname{dim} \mathfrak{g} \gamma_{m n} l_{k}^{m n}
$$


But $\gamma_{m n} l_{k}^{m n} \neq 0$ implies $-3 g \leq k \leq 0$. Hence for $k$ outside of this range the order of $(m, n)$ is of no importance. In particular we can always take the order corresponding to the normal ordering $\Sigma_{0}$. Note also that $l_{k}^{n m} \neq 0$ implies $-g \leq k-(n+m) \leq 0$. Hence if $k>0$ either $n$ or $m>0$ for the terms where $l_{k}^{n m} \neq 0$. By this and (5-1) we get $L_{k}^{*} v=0$ in this case, hence (5-9). For $k<-3 g$ we see that only pairs $(n, m)$ with $n, m \leq 0$ will occur. Again $l_{k}^{n m} \neq 0$ implies that $(n+m)<-2 g$ which could only be the case if $n$ or $m$ is less than $-g$. Now (4), (5) and the almost-grading of the algebra $A$ implies that the resulting vector $\sum_{i} u_{i}(m) u^{i}(n) v$ will have only elements of degree $<\operatorname{deg} v$. Hence the claim for $L_{k}^{*} v$ for $k<-3 g$. The equation $(5-10)$ is automatic because $v$ is the element of highest degree. Note that :...: was allowed to be an arbitrary normal ordering.

Using the arguments in the proof above we see that with respect to the normal ordering (5-8) the $\operatorname{deg} v$-part of $L_{k}^{*}$ for $-3 g \leq k \leq 0$ will only come from

$$
L_{k}^{*} v=\frac{1}{2} \sum_{-g \leq m, n \leq 0} l_{k}^{m n}: u_{i}(m) u^{i}(n): v+\ldots
$$

For more general normal orderings an additional term $M(k, \Sigma) v$ will occur where $M(k, \Sigma)$ is a scalar depending on the normal ordering.

By definition $\lambda=\left(\lambda_{0}, \lambda_{-1}, \ldots, \lambda_{-3 g}\right)$ with the $\lambda_{i}$ from Lemma 5.1 is the weight of the Sugawara representation. As usual we denote by $\Lambda$ the corresponding quadratic differential.

Lemma 5.2. Let $T$ be the Sugawara operator (or energy-momentum tensor) as introduced by (3-3) then

$$
T . v=-(\mathrm{c}+\mathrm{k}) \Lambda \cdot v+\ldots .
$$

Note that T.v is a formal sum. But if we decompose it into its homogeneous parts with respect to the degree in $V$ then the components are well-defined elements of $V \otimes \mathcal{F}^{2}$ ( $\mathcal{F}^{2}$ is the space of quadratic differentials). The Equation (5-11) should be interpreted in this sense.

Proof of Lemma 5.2.

$$
T . v=\sum_{k \in \mathbb{Z}} \Omega^{k} L_{k} v=\sum_{k \leq 0} \Omega^{k} L_{k} v .
$$

By isolating the highest degree part using Lemma 5.1 we see

$$
(T . v)_{h . D .}=-(\mathrm{c}+\mathrm{k}) \sum_{k=-3 g}^{0} \Omega^{k} L_{k}^{*} v=-(\mathrm{c}+\mathrm{k}) \sum_{k=-3 g}^{0} \Omega^{k} \lambda_{k} v=-(\mathrm{c}+\mathrm{k}) \Lambda \cdot v \text {. }
$$


The following theorem answers the question which was put in the beginning of this section. The analogue of this theorem for the less complicated case of Heisenberg type algebras (which corresponds to abelian $\mathfrak{g}$ ) was proved in [20].

Theorem 5.1. Let $\chi$ be the highest weight of the $\widehat{\mathcal{G}}$-module $V$ fulfilling the condition (1)-(5), $\Sigma$ be a normal ordering and $\Lambda$ be the weight of the corresponding Sugawara representation of $\widehat{\mathcal{L}}$. Then the following relations for $\Lambda$ hold:

$$
\Lambda=\frac{-1}{\mathrm{c}+\mathrm{k}} \sum_{-g \leq m, n \leq 0}\left(\frac{1}{2}<\chi_{m}, \chi_{n}>+\sum_{-g \leq s \leq 0} \alpha_{m n}^{s}<\chi_{s}, \bar{\rho}>-\mathrm{p} \cdot \mathrm{c} \gamma_{[m n]}\right) \omega^{m} \omega^{n}
$$

and

$$
\Lambda=\frac{-1}{\mathrm{c}+\mathrm{k}} \sum_{-g \leq m, n \leq 0}\left(\frac{1}{2}<\chi_{m}, \chi_{n}>+\sum_{-g \leq s \leq 0} \alpha_{m n}^{s}<\chi_{s}, \bar{\rho}>\right) \omega^{m} \omega^{n}+K(\Sigma),
$$

with

$$
K(\Sigma):=\frac{\mathrm{p} \cdot \mathrm{c}}{\mathrm{c}+\mathrm{k}} \cdot\left(\sum_{(m, n) \in \Sigma_{c s}^{+}}-\sum_{(m, n) \in \Sigma_{c s}^{-}}\right) \gamma_{m n} \omega^{m} \omega^{n} .
$$

Here the $\alpha_{m n}^{s}$ are the structure constants of the associative algebra $\mathcal{A}$ defined by (2-13), $\gamma_{[m n]}:= \pm \gamma_{m n}$ if $(m, n) \in \Sigma^{ \pm}$respectively, $2 \bar{\rho}=\sum_{\alpha>0} \alpha$ is the sum over the positive roots, and $p$ is the number of positive roots, i.e. $p=1 / 2(\operatorname{dim} \mathfrak{g}-\operatorname{rank} \mathfrak{g})$.

For a normal ordering $\Sigma$ with $\Sigma_{c s}^{+}$(or $\left.\Sigma_{c s}^{-}\right) \subseteq\{(m, n) \mid-g \leq m, n \leq 0\}$ we obtain $K(\Sigma)=0$.

Proof. By Lemma 5.2 it is enough to find the term of highest degree in T.v, where $T$ is given by (3-2). Note that by $(4),(5): u_{i}(m) u^{i}(n): v$ has a nonzero projection onto $\mathbb{C} v$ only if $-g \leq n, m \leq 0$. So

$$
T . v=\left(\frac{1}{2} \sum_{-g \leq m, n \leq 0} \sum_{i}: u_{i}(m) u^{i}(n): \omega^{m} \omega^{n}\right) v+\ldots
$$

For the rest of the proof let $-g \leq m, n \leq 0$. We choose as basis in $\mathfrak{g}$ the "canonical basis". Choose $h_{k}, k=1, \ldots$, rank $\mathfrak{g}$ for the Cartan subalgebra and $h^{k}, k=1, \ldots$, rank $\mathfrak{g}$ as dual basis with respect to the invariant form (..|..). Choose for the positive roots $\alpha$ basis elements $x_{\alpha}$ and take as dual $x_{-\alpha}$ the rescaled element of the corresponding negative root (e.g. $\left.\left(x_{\alpha} \mid x_{-\alpha}\right)=1\right)$. With respect to this basis $\left(h_{k}, x_{\alpha}, x_{-\alpha}\right)$

$$
\sum_{i} u_{i}(m) u^{i}(n)=\sum_{\alpha>0}\left(x_{\alpha}(m) x_{-\alpha}(n)+x_{-\alpha}(m) x_{\alpha}(n)\right)+\sum_{k} h_{k}(m) h^{k}(n),
$$


where $k=1, \ldots$, rank $\mathfrak{g}$ and $\alpha$ runs over all positive roots. Using the structure equation of $\widehat{\mathcal{G}}$ we can write

$$
\begin{aligned}
x_{\alpha}(m) x_{-\alpha}(n)=x_{-\alpha}(n) x_{\alpha}(m)+ & {\left[x_{\alpha}(m), x_{-\alpha}(n)\right]=} \\
& =x_{-\alpha}(n) x_{\alpha}(m)+\sum_{s} \alpha_{m n}^{s} h_{\alpha}(s)-\gamma_{m n} t,
\end{aligned}
$$

where $h_{\alpha}:=\left[x_{\alpha}, x_{-\alpha}\right]$ and the $\gamma_{m n}$ are defined by (3-8). We replace the summands in $(5-15)$ at the first position by $(5-16)$. Now $\left(x_{-\alpha}(m) x_{\alpha}(n)\right) v=0 \cdot v+\ldots$, by $(5-4)$, hence there remains only

$$
\sum_{i} u_{i}(m) u^{i}(n) v=\left(\sum_{k} h_{k}(m) h^{k}(n)+\sum_{s} \sum_{\alpha>0} \alpha_{m n}^{s} h_{\alpha}(s)-p \cdot \gamma_{m n} t\right) v+\ldots
$$

If $(m, n) \in \Sigma_{c s}^{+}$then this is already in normal order. Note that $h_{\alpha}(s) v$ will have a $v$-component only if $-g \leq s \leq 0$. Now by (5-2), (5-3) we obtain

$$
\left(\sum_{k} \chi_{m}\left(h_{k}\right) \chi_{n}\left(h^{k}\right)+\sum_{s=-g}^{0} \sum_{\alpha>0} \alpha_{m n}^{s} \chi_{s}\left(h_{\alpha}\right)-p \cdot \gamma_{m n} \mathrm{c}\right) v+\ldots .
$$

For the pairs $(m, n) \in \Sigma_{c s}^{-}$we get

$$
\begin{aligned}
\sum_{i}: u_{i}(m) u^{i}(n): v & =\sum_{i} u^{i}(n) u_{i}(m) v \\
& =\left(\sum_{k}: h_{k}(m) h^{k}(n):+\sum_{s} \sum_{\alpha>0} \alpha_{n m}^{s} h_{\alpha}(s)-p \cdot \gamma_{n m} t\right) v+\ldots \\
& =\left(\sum_{k} \chi_{m}\left(h_{k}\right) \chi_{n}\left(h^{k}\right)+\sum_{s=-g}^{0} \sum_{\alpha>0} \alpha_{m n}^{s} \chi_{s}\left(h_{\alpha}\right)+p \cdot \gamma_{m n} \mathrm{c}\right) v+\ldots,
\end{aligned}
$$

where we used $\alpha_{m n}^{s}=\alpha_{n m}^{s}$ and $\gamma_{m n}=-\gamma_{n m}$.

Let us denote the dual bilinear form on $\mathfrak{h}^{*}$ by $<\ldots, . .>$. We use

$$
\sum_{k} \chi_{m}\left(h_{k}\right) \chi_{n}\left(h^{k}\right)=<\chi_{m}, \chi_{n}>, \quad \chi_{s}\left(h_{\alpha}\right)=<\chi_{s}, \alpha>, \quad \text { and } \quad \sum_{\alpha>0} \alpha=: 2 \bar{\rho},
$$

in (5-18) and (5-19) and obtain after summation over $n$ and $m$

$$
\begin{aligned}
T v= & \left(\frac{1}{2} \sum_{-g \leq m, n \leq 0} \sum_{i}: u_{i}(m) u^{i}(n): \omega^{m} \omega^{n}\right) v+\ldots \\
= & \sum_{-g \leq m, n \leq 0}\left(\frac{1}{2}<\chi_{m}, \chi_{n}>+\sum_{-g \leq s \leq 0} \alpha_{m n}^{s}<\chi_{s}, \bar{\rho}>-p \cdot c \gamma_{[m n]}\right) \omega^{m} \omega^{n} v+\ldots \\
= & \sum_{-g \leq m, n \leq 0}\left(\frac{1}{2}<\chi_{m}, \chi_{n}>+\sum_{s=-g}^{0} \alpha_{m n}^{s}<\chi_{s}, \bar{\rho}>\right) \omega^{m} \omega^{n} v-(\mathrm{c}+\mathrm{k}) K(\Sigma) v+\ldots
\end{aligned}
$$


After dividing this by $-(\mathrm{c}+\mathrm{k})$ we obtain equations (5-12) and (5-13). For the special normal ordering $\Sigma$ in the theorem we have $\Sigma_{c s}^{-}=\emptyset$ and $\Sigma_{c s}^{+}$is the full square. For $(m, n)$ with $m \neq n$ also the pair $(n, m)$ will appear. Because $\gamma_{n m}=-\gamma_{m n}$ they will cancel. $\gamma_{m m}$ will always be zero. Hence, $K(\Sigma)=0$. The same is true if we interchange the role of $\Sigma_{c s}^{+}$and $\Sigma_{c s}^{-}$.

Note again that a more general normal ordering will yield that the summation prescription in the definition of $K(\Sigma)$ will be different.

Example. Let $g=0$. Then (5-12) specializes to

$$
\Lambda=\frac{-1}{\mathrm{c}+\mathrm{k}}\left(\frac{1}{2}<\chi_{0}, \chi_{0}>+<\chi_{0}, \bar{\rho}>\right)\left(\omega^{0}\right)^{2},
$$

where $\omega^{0}=\frac{1}{z} \mathrm{~d} z$. Note that in this case $K(\Sigma)$ will always vanish. Up to the factor $\frac{-1}{2(c+k)}\left(\omega^{0}\right)^{2}$ this expression coincides with the eigenvalue of Casimir operator of second order for $\mathfrak{g}$.

\section{The Casimir operator and its eigenvalues}

As it is pointed out in [8, Lecture 10] the Sugawara construction is closely related to the Casimir operators of Kac-Moody algebras. In this section generalizing the approach of [8] we obtain a Casimir operator of the second order and under certain assumptions its eigenvalues for an arbitrary Krichever-Novikov algebra of affine type.

In order to do this we first extend our algebra $\widehat{\mathcal{G}}$ by adjoining an arbitrary vector field $e \in \mathcal{L}$. As vector space we take $\widehat{\mathcal{G}}^{e}=\widehat{\mathcal{G}} \oplus \mathbb{C} \cdot e$ and define

$$
\left[e, x \otimes A_{n}\right]=x \otimes \nabla_{e} A_{n}, \quad[e, t]=0 .
$$

Proposition 6.1. $\quad \widehat{\mathcal{G}}^{e}$ is a Lie algebra.

Proof. We have to show the Jacobi identity for commutators in which the new element $e$ is involved, i.e. for the triples $(e, x(n), e)$, here it is clear, and $(e, x(n), y(m))$, here we shall show it. Now

$$
\begin{aligned}
{[e,[x(n), y(m)]] } & =\left[e,[x, y] \otimes A_{n} A_{m}-(x \mid y) \gamma\left(A_{n}, A_{m}\right) t\right]=[x, y] \otimes \nabla_{e}\left(A_{n} A_{m}\right) \\
& \left.=[x, y] \otimes\left(\left(\nabla_{e} A_{n}\right) A_{m}\right)+A_{n}\left(\nabla_{e} A_{m}\right)\right) \\
{[x(n),[y(m), e]] } & =-\left[x(n), y \otimes \nabla_{e} A_{m}\right]=-[x, y] \otimes A_{n} \nabla_{e} A_{m}+(x \mid y) \gamma\left(A_{n}, \nabla_{e} A_{m}\right) t \\
{[y(m),[e, x(n)]] } & =\left[y(m), x \otimes \nabla_{e} A_{n}\right]=-[x, y] \otimes A_{m} \nabla_{e} A_{n}-(y \mid x) \gamma\left(A_{m}, \nabla_{e} A_{n}\right) t .
\end{aligned}
$$


We have to show that the central terms will cancel:

$$
\begin{aligned}
& \gamma\left(A_{n}, \nabla_{e} A_{m}\right)=\frac{1}{2 \pi \mathrm{i}} \int_{C_{\tau}} A_{n} d\left(\nabla_{e}\left(A_{m}\right)\right)=\frac{1}{2 \pi \mathrm{i}} \int_{C_{\tau}} A_{n} \nabla_{e}\left(d A_{m}\right) \\
= & \frac{1}{2 \pi \mathrm{i}} \int_{C_{\tau}} \nabla_{e}\left(A_{n} d A_{m}\right)-\frac{1}{2 \pi \mathrm{i}} \int_{C_{\tau}}\left(\nabla_{e} A_{n}\right) d A_{m}=\frac{1}{2 \pi \mathrm{i}} \int_{C_{\tau}} A_{m} d\left(\nabla_{e}\left(A_{n}\right)\right)=\gamma\left(A_{m}, \nabla_{e} A_{n}\right) .
\end{aligned}
$$

Here we used that the Lie derivative commutes with the exterior differentiation, that it is a derivation, and that the Lie derivative of a meromorphic form has no residue (see $[16, \mathrm{p} .102])$.

Note that in the situation of zero genus [7] one takes $e=z \frac{d}{d z}=d$ and obtains for (6-1) $[e, x(n)]=n \cdot x(n)$.

Let the vector field $e$ be fixed and decomposed as $e=\sum_{k} \varepsilon^{k} e_{k}$ (a finite sum). Let $V$ be a highest weight representation of $\widehat{\mathcal{G}}$ which extends to a highest weight representation of $\widehat{\mathcal{G}}^{e}$. In what follows we do not distinguish between $e$ as element of the Lie algebra $\widehat{\mathcal{G}}^{e}$ and the corresponding operator on the representation space. The weight $\lambda_{e}$ corresponding to $e$ is given by $e \cdot v=\lambda_{e} v+\ldots$

Theorem 6.1. Let $e=\sum_{k} \varepsilon^{k} e_{k}$ be the vector field used to define the algebra $\widehat{\mathcal{G}}^{e}$. Let the finite dimensional algebra $\mathfrak{g}$ be either simple or abelian. Let $V$ be a highest weight representation of $\widehat{\mathcal{G}}^{e}$ and $L=\sum_{k} \varepsilon^{k} L_{k}$ be the corresponding linear combination of the Sugawara operators $L_{k}$ introduced in Section 3, then

$$
\Omega:=2 L+2(\mathrm{c}+\mathrm{k}) e
$$

is a Casimir operator of $\widehat{\mathcal{G}}^{e}$ for the representation $V$. The number $\mathrm{c}$ is the central charge (i.e. $t . v=\mathrm{c} \cdot v$ ) and $\mathrm{k}$ is the dual Coxeter number (resp. 0 in the abelian case) introduced in Lemma 3.1.

Proof. We have to show that $\Omega$ commutes with all other operators of $\widehat{\mathcal{G}}^{e}$.

(1) Note that we have $[e, x(n)]=x\left(\nabla_{e} A_{n}\right)$. On the other hand by Prop 3.2(1)

$$
[L, x(n)]=-(\mathrm{c}+\mathrm{k}) \sum_{k} \varepsilon^{k}\left[L_{k}, x(n)\right]=-(\mathrm{c}+\mathrm{k}) \sum_{k} \varepsilon^{k} x\left(\nabla_{e_{k}} A_{n}\right)=-(\mathrm{c}+\mathrm{k}) x\left(\nabla_{e} A_{n}\right)
$$

Hence $[\Omega, x(n)]=0$.

(2) It remains to show $[\Omega, e]=0$. First we want to calculate $\left[e, L_{k}\right]$. Due to the linearity of the Lie derivative and $(6-1)$ the operator $E:=-(c+k) e$ obeys the relation

$$
[E, x(n)]=-(\mathrm{c}+\mathrm{k}) x\left(\nabla_{e} A_{n}\right)=[L, x(n)]
$$


by Prop. 3.2(1). Hence from Corollary 4.1 we get $\left[L_{k}, E\right]=\left[L_{k}, L\right]$. Now

$$
-(\mathrm{c}+\mathrm{k})[L, e]=-(\mathrm{c}+\mathrm{k}) \sum_{k} \varepsilon^{k}\left[L_{k}, e\right]=\sum_{k} \varepsilon^{k}\left[L_{k}, L\right]=[L, L]=0 .
$$

Because $(c+k) \neq 0$ this implies $[L, e]=0$ and further $[\Omega, e]=0$.

In the remainder of the section let us discuss the question of eigenvalues of the Casimir operators. In zero genus case a Casimir operator acts as multiplication by a scalar on the highest weight module. The usual argument is that the highest weight vector $v$ is an eigenvector of the Casimir operator: $\Omega v=\lambda v[8]$. Each other element $w$ of this module can be represented in the form $w=u v$ where $u$ belongs to the universal enveloping algebra (cf.(5-1)). As $\Omega$ commutes with $u$ one has $\Omega w=\Omega u v=u \Omega v=\lambda u v=\lambda w$. This argument does not work in the almost-graded situation. In general the question whether $\Omega$ has even at least one eigenvector has no obvious answer. Indeed in the case that the highest weight vector $v$ is an eigenvector of the Casimir operator $\Omega$ one can conclude as above that $\Omega$ operates as a scalar. This scalar can be found in the following way. Recall the definition of the complex number $\lambda_{e}$ by the relation $e . v=\lambda_{e} v+\ldots$.

Proposition 6.1. Let $\varepsilon^{k}=0$ for $k<-g$ or $k>0$. Suppose that the Casimir operator $\Omega$ as defined in (6-2) acts in a highest weight representation of weight $\chi=$ $\left(\chi_{0}, \chi_{-1}, \ldots, \chi_{-g}\right)$ as multiplication by a constant $\lambda_{\Omega}$. Then this constant equals

$$
\lambda_{\Omega}=\sum_{k, m, n} \varepsilon^{k} l_{k}^{m n}\left(<\chi_{m}, \chi_{n}>+2 \sum_{s} \alpha_{m n}^{s}<\chi_{s}, \bar{\rho}>-2 p \cdot c \gamma_{[m n]}\right)+2(c+k) \lambda_{e},
$$

(where all summations are over the range $-g, \ldots,-1,0)$. The term with $\gamma_{[m n]}$ will vanish if a normal ordering $\Sigma$ is chosen such that $K(\Sigma)=0$.

Proof. It follows from the Theorem 5.1 that the following relation for the highest weight of the Sugawara representation holds:

$$
-(\mathrm{c}+\mathrm{k}) \cdot 2 \Lambda=\sum_{m, n}\left(<\chi_{m}, \chi_{n}>+2 \sum_{s} \alpha_{m n}^{s}<\chi_{s}, \bar{\rho}>-2 p \cdot c \gamma_{[m n]}\right) \omega^{m} \omega^{n} .
$$

On the left-hand side we use the definition (5-5) $\Lambda=\sum_{k=-3 g}^{0} \lambda_{k} \Omega^{k}$ and on the right hand side $\omega^{m} \omega^{n}=\sum l_{k}^{m n} \Omega^{k}$ (see $\left.(3-4)\right)$. If we compare both sides we obtain

$$
-(\mathrm{c}+\mathrm{k}) 2 \lambda_{k}=\sum_{m, n} l_{k}^{m n}\left(<\chi_{m}, \chi_{n}>+2 \sum_{s} \alpha_{m n}^{s}<\chi_{s}, \bar{\rho}>-2 p \cdot c \gamma_{[m n]}\right) .
$$

From the definition of $\Omega=2 L+2(\mathrm{c}+\mathrm{k}) e$ with $L=\sum_{k} \varepsilon^{k} L_{k}=-(\mathrm{c}+\mathrm{k}) \sum_{k} \varepsilon^{k} L_{k}^{*}$ we obtain $\Omega v=\lambda_{\Omega} v$, where $\lambda_{\Omega}$ is given as the expression (6-3).

Again, for more general normal orderings $\Sigma$ an explicit correction term can be given. 
Example. Let $g=0$. Then the only admissible values of $k, m, n$ are $k=m=n=0$, and we have $l_{0}^{00}=\alpha_{00}^{0}=1$. Set $e=z \frac{d}{d z}=d$ (i.e. $\varepsilon^{0}=1$ ) then the result of Theorem 6.2 specializes to

$$
\lambda_{\Omega}=<\chi_{0}+2 \bar{\rho}, \chi_{0}>+2(c+k) \lambda_{e} .
$$

This is the as expected the result, as it can be found (for example) in [8, Prop.10.2] $\lambda_{\Omega}=<\tilde{\chi}+2 \rho, \tilde{\chi}>$, where $\tilde{\chi}$ is the weight of the module $V$ (including the central charge and the vector field $\mathrm{d}$ ) and $\rho$ is the sum of fundamental weights for the affine Kac-Moody algebra $\widehat{\mathcal{G}}^{e}$.

\section{Appendix: Sugawara CONSTRUCtion fOR the MUlTi-POINT SITUATION}

The Sugawara construction above can be generalized to the situation where one allows poles at more than two points. The definition of $\mathcal{F}^{\lambda}, \mathcal{A}, \mathcal{L}, \mathcal{G}$ is completely analogous to the definition in Section 2. The crucial step is to introduce an almost-grading and to find dual systems of basis elements. This is done in [16], [15, 3.ref.]. For a quick review see [17]. (In this context see also Sadov [13] and the appendix of [12].) We want to recall here some steps. Let $A$ be the finite set of points where poles are allowed (which for $g \geq 1$ are in generic position). The set $A$ has to be splited into two non-empty disjoint subset $I$ and $O$. The set $I$ is called the set of in-points, the set $O$ the set of out-points. Let $\# K$ be the number of in-points. One fixes again a set of basis elements $f_{n, p}^{\lambda}, n \in \mathbb{Z}, p=1, \ldots, K$ of $\mathcal{F}^{\lambda}$ (the space of meromorphic forms of weight $\lambda$ which are holomorphic on $\Gamma \backslash A$ ), by requiring certain zero orders at the points of $A$. To give an example: Let the number of in-points be equal to the number of out-points. $\lambda \neq 0,1$, and $I=\left\{P_{1}, P_{2}, \ldots, P_{K}\right\}, O=\left\{Q_{1}, Q_{2}, \ldots, Q_{K}\right\}$ be points in generic positions. Then there is for every $n \in \mathbb{Z}$ and every $p=1, \ldots, K$ up to multiplication with a scalar a unique element $f_{n, p}^{\lambda} \in \mathcal{F}^{\lambda}$ with

$$
\begin{aligned}
\operatorname{ord}_{P_{i}}\left(f_{n, p}^{\lambda}\right) & =(n+1-\lambda)-\delta_{i, p}, \quad i=1, \ldots, K, \\
\operatorname{ord}_{Q_{i}}\left(f_{n, p}^{\lambda}\right) & =-(n+1-\lambda), \quad i=1, \ldots, K-1, \\
\operatorname{ord}_{Q_{K}}\left(f_{n, p}^{\lambda}\right) & =-(n+1-\lambda)+(2 \lambda-1)(g-1) .
\end{aligned}
$$

This can be shown either by using Riemann-Roch type arguments or by explicit constructions. These elements will be the basis elements. In the general situation there are modifications only at the out-points. The element $f_{n, p}^{\lambda}$ is defined to be a homogeneous element of degree $n$. One fixes a differential $\rho \in \mathcal{F}^{1}$ which has exact pole order 1 at the points in $A$, positive residues at the points in $I$, negative residues at the points in $O$, and purely imaginary periods. The level lines $C_{\tau}$ are defined completely in the same 
manner as in (2-1). Every level line separates the in- from the out-points. For $\tau \ll 0$ the level line $C_{\tau}$ is a disjoint union of deformed circles around the points in $I$. For $\tau \gg 0$ it is a disjoint union of deformed circles around the points in $O .{ }^{4}$ We obtain the important duality

$$
\frac{1}{2 \pi \mathrm{i}} \int_{C_{\tau}} f_{n, p}^{\lambda} \cdot f_{m, r}^{1-\lambda}=\delta_{n,-m} \cdot \delta_{p, r}
$$

Again we use $A_{n, p}=f_{n, p}^{0}, e_{n, p}=f_{n, p}^{-1}, \omega^{n, p}=f_{-n, p}^{1}, \Omega^{n, p}=f_{-n, p}^{2}$. One obtains an almost-graded structure with respect to the above introduced degree. Analogous formulas to (2-13) and (2-14) are valid. For example

$$
A_{n, p} \cdot A_{m, r}=\sum_{k=n+m}^{n+m+L} \sum_{s=1}^{K} \alpha_{(n, p),(m, r)}^{(k, s)} A_{k, s}, \alpha_{(n, p),(m, r)}^{(k, s)}=\frac{1}{2 \pi \mathrm{i}} \int_{C_{\tau}} A_{n, p} A_{m, r} \omega^{k, s}
$$

Note that we even have $\alpha_{(n, p)(m, r)}^{(n+m, s)}=\delta_{p}^{s} \cdot \delta_{r}^{s}$. Of course the constant $L$ (and $M$ in the equivalent formula to (2-13) and all other introduced bounds) depend also on the number of points in $A$ and their splitting into $I$ and $O$. Explicit formulas can be found in [16]. The corresponding "delta distribution" is now

$$
\Delta\left(Q^{\prime}, Q\right)=\sum_{n \in \mathbb{Z}} \sum_{p=1}^{K} A_{n, p}\left(Q^{\prime}\right) \omega^{n, p}(Q)
$$

For $\alpha \in H^{1}(\Gamma \backslash A, \mathbb{Z})$ the 2-cocycles $(2-3)$ and (2-6) define again central extensions $\widehat{\mathcal{A}}_{\alpha}$ and $\widehat{\mathcal{L}}_{\alpha}$ of $\mathcal{A}$, resp. of $\mathcal{L}$, and Equation (2-5) defines a generalized multi-point affine Kac-Moody algebra $\widehat{\mathcal{G}}_{\alpha}$. If we choose a level line as integration cycle the cocycles will be local, i.e. we have similar bounds as in (2-15). Hence by defining $\operatorname{deg}(t)=0$ we are able to extend the almost-grading to the centrally extended algebras $\widehat{\mathcal{A}}, \widehat{\mathcal{L}}, \widehat{\mathcal{G}}$. Again, without mentioning the cycle $\alpha$ we mean integration along a level line $C_{\tau}$.

We have a decomposition $\mathcal{A}=\mathcal{A}_{-} \oplus \mathcal{A}_{0} \oplus \mathcal{A}_{+}$, and $\mathcal{G}=\mathcal{G}_{-} \oplus \mathcal{G}_{0} \oplus \mathcal{G}_{+}$induced from $\mathcal{A}_{-}:=\left\langle A_{n} \mid n \leq-P-1\right\rangle, \mathcal{A}_{0}:=\left\langle A_{n} \mid-P \leq n \leq 0\right\rangle, \mathcal{A}_{+}:=\left\langle A_{n} \mid n \geq 1\right\rangle$, where $P$ is now a suitable positive constant. Everything works as above. For $x \in \mathfrak{g}$ we define $x(n, p):=x \otimes A_{n, p} \in \widehat{\mathcal{G}}$. Hence the (non-central) generators of $\widehat{\mathcal{G}}$ come with two labels. Let $V$ be an admissible representation of $\widehat{\mathcal{G}}$ then we denote by $x(A)$ the operator corresponding to the element $x \otimes A$. we use also $x(n, p):=x\left(A_{n, p}\right)$.

Where we had above a summation over $n$ we have to add to this summation another (finite) summation over $p=1, \ldots, K: \widehat{u^{i}}(Q)=\sum_{n} \sum_{p} u^{i}(n, p) \cdot \omega^{n, p}(Q)$. Here and in the following the summation over the first index of the label is always over $\mathbb{Z}$ and over

\footnotetext{
${ }^{4}$ In the interpretation of string theory this $\tau$ might be interpreted as proper time of the string on the world sheet.
} 
the second index of the label over $1, \ldots, K$ if nothing else is said. The higher genus (multi-point) Sugawara operator is defined as

$$
T(Q):=\frac{1}{2} \sum_{i}: \widehat{u_{i}}(Q) \widehat{u^{i}}(Q):=\frac{1}{2} \sum_{n, m} \sum_{p, s} \sum_{i}: u_{i}(n, p) u^{i}(m, s): \omega^{n, p}(Q) \omega^{m, s}(Q) .
$$

We decompose it again as

$$
T(Q)=\sum_{k} \sum_{r} L_{k, r} \cdot \Omega^{k, r}(Q)
$$

with

$$
\begin{aligned}
L_{k, r}=\frac{1}{2 \pi \mathrm{i}} \int_{C_{\tau}} T(Q) e_{k, r}(Q) & =\frac{1}{2} \sum_{n, m} \sum_{p, s} \sum_{i}: u_{i}(n, p) u^{i}(m, s): l_{(k, r)}^{(n, p)(m, s)}, \\
\text { where } \quad l_{(k, r)}^{(n, p)(m, s)} & =\frac{1}{2 \pi \mathrm{i}} \int_{C_{\tau}} w^{n, p}(Q) w^{m, s}(Q) e_{k, r}(Q) .
\end{aligned}
$$

We define $\gamma_{(n, p)(m, s)}:=\gamma\left(A_{n, p}, A_{m, s}\right):=\frac{1}{2 \pi \mathrm{i}} \int_{C_{\tau}} A_{n, p}(Q) d A_{m, s}(Q)$, and obtain

Proposition A.1. Let $\mathfrak{g}$ be either an abelian or a simple Lie algebra, then we have

$$
\begin{gathered}
{\left[L_{k, r}, x(n, p)\right]=-(\mathrm{c}+\mathrm{k}) \sum_{m} \sum_{s} K_{(n, p),(k, r)}^{(m, s)} x(m, s)} \\
{\left[L_{k, r}, x(n, p)\right]=-(\mathrm{c}+\mathrm{k}) x\left(\nabla_{e_{k, r}} A_{n, p}\right),} \\
{\left[L_{k, r}, \widehat{x}(Q)\right]=(\mathrm{c}+\mathrm{k}) e_{k, r} . \widehat{x}(Q),} \\
\text { with } K_{(n, p),(k, r)}^{(m, s)}=\frac{1}{2 \pi \mathrm{i}} \int_{C_{\tau}} w^{m, s} e_{k, r} d A_{n, p}=\sum_{l} \sum_{v} l_{(k, r)}^{(m, s)(l, v)} \gamma_{(l, v)(n, p)} \cdot
\end{gathered}
$$

The result does not depend on the normal ordering.

If one checks the proof in Section 4 one sees that essentially only the duality and the "delta distribution" has been used and they have been generalized. Beside this one uses also the generalization of the Lemma of Bonora et al.:

Lemma A.1. For every $N$

$$
\left(\sum_{\substack{n>N \\ m \leq N}}-\sum_{\substack{m>N \\ n \leq N}}\right) \sum_{p, s} A_{n, p}\left(Q^{\prime}\right) A_{m, s}(Q) \omega^{m, s}\left(Q^{\prime}\right) \omega^{n, p}(Q)=d^{\prime} \Delta\left(Q^{\prime}, Q\right)
$$


Here $d^{\prime}$ means differentiation with respect to the variable $Q^{\prime}$.

Sketch of the proof. In the appendix of [12] Krichever and Novikov state the uniqueness of a local 2-cocycle for the (multi-point) algebras $\mathcal{A}$ (up to scalar) and $\mathcal{L}$ (up to scalar and coboundary). Indeed the proof of the "uniqueness" presented in [10] for the 2-point situation using discrete Baker-Akhiezer functions should generalize (see [16, p.89]). If we use this claim we can argue as in Section 4. The right hand side of (4-11) in its suitable generalization has to be again a scalar multiple of the cocycle (2-3) (with integration over a level line). To fix the scalar we calculate $\left[A_{i, p}, A_{-i, p}\right] \Phi$. Note that we have $A_{k, s} A_{n, r}=A_{k+n, r} \delta_{s}^{r}+A$-terms with higher degree as $k+n$ and $\gamma\left(A_{i, p}, A_{-i, p}\right)=(-i)$ (there is only a residue at the point $P_{p}$ ). If we want to avoid the statement about the uniqueness we have to make lengthy local calculations as indicated in [1].

Now Proposition 3.3 and its proof have their obvious generalizations

Proposition A.2. The operators $L_{k} \in g l(V)$ and $i d=1 \in g l(V)$ close up to a Lie subalgebra of $g l(V)$ with the commutator relation

$$
\left[L_{k, r}, L_{l, v}\right]=-(\mathrm{c}+\mathrm{k}) \sum_{n} \sum_{p} C_{(k, r)(l, v)}^{(n, p)} L_{n, p}-\frac{1}{2} \mathrm{c}(\mathrm{c}+\mathrm{k}) \operatorname{dim} \mathfrak{g} \cdot \chi_{(k, r)(l, v)} \cdot i d .
$$

$\chi_{(k, r)(l, v)} \neq 0$ implies that $-P \leq k+l \leq 0$ with a positive constant $P$ not depending on $k$ and $l$.

The splitting of $\chi_{(k, r)(l, s)}$ and its expression is completely analogous to (3-11). The same is true for the expression (3-12) for the form of the cocycle for certain basis elements.

We get the

Theorem A.1. Let $\mathfrak{g}$ be either a finite dimensional abelian or simple Lie algebra and $2 \mathrm{k}$ be the eigenvalue of the Casimir operator in the adjoint representation. Let $A$ be a finite set of points (for $g \geq 1$ in general position) and $A=I \cup O$ a splitting into two disjoint non-empty subsets. Let $\mathcal{A}$ be the algebra of meromorphic functions which are holomorphic on $\Gamma \backslash A$ and $\widehat{\mathcal{G}}$ be the generalized higher genus multi-point affine Kac-Moody algebra, i.e. the central extension of $\mathcal{G}=\mathfrak{g} \otimes \mathcal{A}$ defined by

$$
\widehat{[x \otimes f}, \widehat{y \otimes g}]=[x, \widehat{y] \otimes(} f g)-(x \mid y) \cdot \frac{1}{2 \pi \mathrm{i}} \int_{C_{\tau}} f d g \cdot t, \quad\left[t, \widehat{\mathcal{G}}_{\alpha}\right]=0,
$$

where $C_{\tau}$ is a level line separating the points in $I$ and $O$. Let $V$ be an admissible representation where the central element $t$ operates as $\mathrm{c} \cdot$ identity. If $\mathrm{c}+\mathrm{k} \neq 0$ then the rescaled modes

$$
L_{k, r}^{*}=\frac{-1}{2(\mathrm{c}+\mathrm{k})} \sum_{n, m} \sum_{p, s} \sum_{i}: u_{i}(n, p) u^{i}(m, s): l_{(k, r)}^{(n, p)(m, s)},
$$


of the Sugawara operator define a representation of a local central extension $\widehat{\mathcal{L}}^{\prime}$ of the Krichever Novikov vector field algebra $\mathcal{L}$.

Using again the statement of Krichever and Novikov about the uniqueness of a local cocycle, and the corresponding result to Lemma 3.2 (see[16]) we get indeed a representation of $\widehat{\mathcal{L}}$ defined by the cocycle $(3-15)$.

\section{REFERENCES}

1. Bonora L., Rinaldi, M., Russo, J., Wu, K., The Sugawara construction on genus g Riemann surfaces, Phys. Lett. B 208 (1988), 440-446.

2. Borcherds, R.E., Vertex algebras, Kac-Moody algebras, and the monster, Proc. Natl. Acad. Sci. USA 83 (1986), 3086-3071.

3. Bremner, M.R., Universal central extensions of elliptic affine Lie algebras, J. Math. Phys. 35 (1994), $6685-6692$.

4. Bremner, M.R., Four-point affine Lie algebras, Proc. Amer. Math. Soc. 123 (1995), 1981-1989.

5. Frenkel, I., Lepowski, J. Meurman, A., Vertex operator algebras and the monster, Academic Press, Boston, 1988.

6. Jaffe, A., Klimek, S., Lesniewski, A., Representations of the Heisenberg algebra on a Riemann surface, Commun. Math. Phys. 126 (1989), 421-431.

7. Kac, V., Infinite dimensional Lie algebras, Camdridge University Press, Cambridge, 1990.

8. Kac, V.G., Raina, A.K., Highest Weight Representations of Infinite Dimensional Lie Algebras, Adv. Ser. in Math. Physics Vol.2, World Scientific, 1987.

9. Krichever, I.M., private commmunication (1995).

10. Krichever, I.M., Novikov, S.P., Algebras of Virasoro type, Riemann surfaces and structures of the theory of solitons, Funktional Anal. i. Prilozhen. 21 (1987), no. 2, 46; Virasoro type algebras, Riemann surfaces and strings in Minkowski space, Funktional Anal. i. Prilozhen. 21 (1987), no. 4, 47; Algebras of Virasoro type, energy-momentum tensors and decompositions of operators on Riemann surfaces, Funktional Anal. i. Prilozhen. 23 (1989), no. 1, 19-23.

11. Krichever, I.M., Novikov, S.P., Virasoro-Gelfand-Fuks type algebras, Riemann surfaces, operator's theory of closed strings, JGP 5 (1988), $631-661$.

12. Krichever, I.M., Novikov, S.P., Riemannn surfaces, Operator fields, Strings. Analogues of FourierLaurent bases, Physics and mathematics of strings (Brink, Friedan, Polyakov, ed.), World Scientific, 1990, pp. 356-388.

13. Sadov, V.A., Bases on multipunctured Riemann surfaces and interacting strings amplitudes, Commun. Math. Phys. 136 (1991), 585-597.

14. Schlichenmaier, M., An Introduction to Riemann Surfaces, Algebraic Curves and Moduli Spaces, Springer Lecture Notes in Physics, Vol. 322, Springer, New York, 1989.

15. Schlichenmaier, M., Krichever-Novikov algebras for more than two points, Lett. Math. Phys. 19 (1990), 151-165; Krichever-Novikov algebras for more than two points: explicit generators, Lett. Math. Phys. 19 (1990), 327-336; Central extensions and semi-infinite wedge representations of Krichever-Novikov algebras for more than two points, Lett. Math. Phys. 20 (1990), 33-46.

16. Schlichenmaier, M., Verallgemeinerte Krichever-Novikov Algebren und deren Darstellungen, 
PhD. Thesis 1990, Universität Mannheim, Germany.

17. Schlichenmaier, M., Differential operator algebras on compact Riemann surfaces, Generalized Symmetries in Physics (Doebner, H.-D., Dobrev, V.K., Ushveridze, A.G., eds.), World Scientific, Singapore, London, 1994, pp. 425-435.

18. Sheinman, O.K., Elliptic affine Lie algebras, Funktional Anal. i. Prilozhen. 24 (1990), no. 3, 210219; Highest weight modules over certain quasigraded Lie algebras on elliptic curves, Funktional Anal. i. Prilozhen. 26 (1992), no. 3, 203-208; Affine Lie algebras on Riemann surfaces, Funktional Anal. i. Prilozhen. 27 (1993), no. 4, 54-62.

19. Sheinman, O.K., Highest weight modules for affine Lie algebras on Riemann surfaces, Funktional Anal. i. Prilozhen. 28 (1995), no. 1.

20. Sheinman, O.K., Representations of Krichever-Novikov algebras, Topics in topology and mathematical physics (Novikov, S.P., ed.), Amer. Math. Soc., Providence, R.I., U.S.A., 1995.

Martin Schlichenmaier, Department of Mathematics and Computer Science, University of Mannheim D-68131 Mannheim, Germany

E-mail address: schlichenmaier@math.uni-mannheim.de

Oleg K. Sheinman, Independent Moscow University, Ul. Marshala Biryuzova 4, Kor.1, KV.65 Moscow, Russia, 123298

E-mail address: sheinman@landau.ac.ru 